\title{
Gut Microbiota-Derived Inosine From Dietary Barley Leaf Supplementation Attenuates Colitis Through PPARy Signaling Activation
}

\section{Daotong Li}

China Agricultural University

\section{Yu Feng}

China Agricultural University

\section{Meiling Tian}

China Agricultural University

\section{Junfu Ji}

China Agricultural University

Xiaosong Hu

China Agricultural University

\section{Fang Chen ( $\nabla$ chenfangch@sina.com )}

China Agricultural University https://orcid.org/0000-0002-1578-0797

\section{Research}

Keywords: Barley leaf, Colitis, Gut microbiota, Purine metabolite, Mucosal barrier, PPARy, Inosine Posted Date: February 11th, 2021

DOl: https://doi.org/10.21203/rs.3.rs-174442/v1

License: (1) This work is licensed under a Creative Commons Attribution 4.0 International License. Read Full License

Version of Record: A version of this preprint was published at Microbiome on April 5th, 2021. See the published version at https://doi.org/10.1186/s40168-021-01028-7. 


\section{Abstract \\ Background}

Ulcerative colitis is a type of chronic inflammatory bowel disease closely associated with gut microbiota dysbiosis and intestinal homeostasis dysregulation. Barley leaf $(\mathrm{BL})$ has a long history of use in Traditional Chinese Medicine with potential health-promoting effects on intestinal functions. However, its mechanism of action is not yet clear. Here, we explore the potential modulating roles of gut microbial metabolites of $B L$ to protect against colitis and elucidate the underlying molecular mechanisms.

\section{Results}

Using 16S rRNA gene-based microbiota analysis, we first found that dietary supplementation of BL ameliorated dextran sulphate sodium (DSS)-induced gut microbiota dysbiosis. The mechanisms by which BL protected against DSS-induced colitis were resulted from improved intestinal mucosal barrier functions via the activation of peroxisome proliferator-activated receptor (PPAR)y signaling. In addition, metabolomic profiling analysis showed that the gut microbiota modulated BL-induced metabolic reprograming in the colonic tissues particularly by the enhancement of glycolysis process. Notably, dietary BL supplementation resulted in enrichment of microbiota-derived purine metabolite inosine, which could activate PPARy signaling in human colon epithelial cells. Furthermore, exogenous treatment of inosine reproduced the similar protective effects as $B L$ to protect against DSS-induced colitis through improving adenosine $2 A$ receptor $\left(A_{2 A} R\right) / P P A R y$-dependent mucosal barrier functions.

\section{Conclusions}

Overall, our findings suggest that the gut microbiota-inosine- $A_{2 A} R / P P A R y$ axis plays an important role in the maintenance of intestinal homeostasis, which may represent a novel approach for colitis prevention via manipulation of the gut microbial purine metabolite.

\section{Background}

Inflammatory bowel disease is a chronic inflammatory disease of the intestine that includes ulcerative colitis and Crohn's disease [1]. Ulcerative colitis is characterized by the presence of discontinuous lesions that mainly affect the mucosal layer in the cecum and colon [2]. Although it is highly prevalent in the developed countries, recent studies have shown that it is becoming more frequent in the developing countries and this disease has emerged as a worldwide public health challenge [3, 4]. Although the etiology of ulcerative colitis is still not fully understood, multiple genetic and environmental factors have been reported to be involved in the pathogenesis of ulcerative colitis [5]. Patients with ulcerative colitis display decreased expression of peroxisome proliferator-activated receptor (PPAR) $y$ in the colonic epithelium, which may be an important factor for the cause of intestinal dysfunction and chronic 
inflammation [6]. Clinically, PPARy is also a crucial pharmacological target for anti-inflammatory drugs such as 5-aminosalicylic acid and corticosteroids [7]. However, most of the current drug-based interventions are lack of specificity and may have adverse effects [8]. There is therefore an unmet need for developing new therapeutic strategies for the prevention and treatment of colitis.

The gut microbiota, which contains trillions of metabolically active microbes, is an important environmental factor affecting host physiology and shaped by diet [9]. The intestinal mucus and epithelial cell layer is the first line of defense limiting the translocation of potentially harmful antigens [10]. Interestingly, germ free and conventionally raised mice exhibited different properties of colonic mucus layer, which were also observed in mice with different gut microbiota composition. Notably, the different properties of colonic mucus layers could be transmissible to germ free mice through gut microbiota transplantation [11, 12]. Moreover, another study showed that transplantation of the gut microbiota from patients with irritable bowel syndrome could lead to alterations of intestinal motility and barrier functions in germ free mice [13]. These findings suggest that the gut microbiota may serve as a pivotal mediator in the maintenance of intestinal homeostasis and targeting the microbiota could be an effective therapeutic approach for preventing and curing colitis [14].

It has been proposed that the effects of the gut microbiota on the host physiological functions partially depend on small molecules derived from host and microbiota co-metabolism, such as short chain fatty acids, bile acids and indole derivatives, which are greatly influenced by dietary nutrients [15-20]. Inosine, a primary metabolite of adenosine, is an important intracellular purine nucleoside. By acting as functional agonists of the adenosine receptors, inosine is involved in the regulation of many physiological and pathophysiological processes [21]. It should be noted that inosine is also important metabolites that are produced by gut bacterial species. Mager et al. showed that gut microbiome-derived metabolite inosine could enhance the effects of checkpoint blockade immunotherapy via activation of antitumor T cells [22].

Barley leaf $(\mathrm{BL})$, the young grass of the crop barley (Hordeum vulgare $\mathrm{L}$.), is the primary component of a popular green-colored functional drink in Asian countries [23]. The antioxidative properties of BL have been reported in previous studies [24,25]. Moreover, as an herbal-based component of Traditional Chinese Medicine, it is historically recorded to have potential health-promoting properties on intestinal functions. However, whether the gut microbiota-derived metabolites play a part in modulating the beneficial effects of $B L$ on intestinal functions and the underlying mechanism have not been elucidated.

In this study with a mouse colitis model, we demonstrated that BL significantly mitigated disease severity and microbiota dysbiosis. We revealed that the underlying mechanisms were related to the improved mucosal barrier functions via peroxisome proliferator-activated receptor (PPAR)y signaling activation. Antibiotic treatment further demonstrated that the gut microbiota was involved in BL-induced metabolic reprograming in colonic tissues. Additionally, we identified a gut microbiota-derived purine metabolite inosine, which mediated the beneficial effects of $B L$ on intestinal functions through adenosine $2 \mathrm{~A}$ receptor $\left(A_{2 A} R\right) / P P A R y$ signaling. Thus, our data provide new insights into the underlying mechanisms of 
$\mathrm{BL}$ in colitis protection and highlighted the potential application of $\mathrm{BL}$ and inosine for the prevention of ulcerative colitis.

\section{Results}

\section{BL ameliorates colitis symptoms and gut microbiota dysbiosis}

In this study, the beneficial impacts of BL on intestinal functions were examined in dextran sodium sulfate (DSS)-induced colitis mouse model, which is a widely used model exhibiting several characteristics resembling human ulcerative colitis [26]. In this model, the epithelial cell barrier is impaired chemically by DSS administration, leading to the disruption of intestinal homeostasis and dysbiosis of gut microbiota. As shown in Fig. 1a, mice were fed a standard chow diet (CD) or an isocaloric diet where $\mathrm{BL}$ was supplemented at a ratio of 2.5\% (Additional file 2: Table S2). There was no significant difference in the food intake or water intake between control and BL-fed mice (Additional file 1: Fig. S1A and B), suggesting that $B L$ had no adverse effects on the eating and drinking habits. During the colitis induction, supplementation of BL significantly protected against DSS-induced body weight loss, reduced disease activity index (DAl) scores and colon shortening (Fig. 1b-d). Supplementation of BL also significantly protected against DSS-induced intestinal permeability (Fig. 1e). Since inflammatory cytokines are crucial factors during the onset of colitis [27], we further assessed the impact of BL on inflammatory cytokines. BL feeding significantly increased the levels of interleukin (IL)-4 and IL-10, and decreased the level of TNF-a in serum of DSS-treated mice (Additional file 1: Fig. S1C-E). A similar change was also observed in colonic tissues (Additional file 1: Fig. S1F-H). These data indicate that BL effectively alleviates colitis symptoms in DSS-treated mice.

Ulcerative colitis is typically associated with dysbiosis of gut microbiota [14]. To study whether BL could prevent against DSS-induced gut microbiota dysbiosis, we analyzed the gut microbitoa composition by performing a $16 \mathrm{~S}$ rRNA gene amplicon sequencing in the colonic contents. The overall structure of gut microbiota that was investigated by UniFrac-based principal coordinates analysis (PCoA) showed that the CD + DSS group displayed a shift clustering of bacterial composition, which was distinct from either CD or BL group (PERMANOVA, $P<0.001$ ) (Fig. 1f), suggesting that the dysbiosis of gut microbiota was induced by DSS treatment. Notably, BL protected against DSS-induced dysbiosis as evidenced by that the microbiota in $B L+D S S$ group was more closely clustered to $C D$ and $B L$ group relative to $C D+D S S$ group (PERMANOVA, $\mathrm{P}<0.001$ ) (Fig. 1f). Consistently, the a-diversity analysis with Chao and Shannon index revealed that BL significantly prevented DSS-induced decrease in bacterial richness and diversity (Fig. 1g). We then further analyzed the gut bacterial composition at phylum and family levels. BL significantly reduced DSS-induced increase in the relative abundance of Proteobacteria (Wilcoxon ranksum test, $P<0.01$ ) (Fig. 1h). Meanwhile, further analysis at family level revealed that DSS treatment caused a dramatic increase in the abundance of Enterobacteriaceae, a common signature of the gut 
microbiota dysbiosis [28], which was also significantly suppressed by BL supplementation (from $20 \% \pm 4-$ $1.5 \% \pm 0.4 \%$, Wilcoxon rank-sum test, $\mathrm{P}<0.0001$ ) (Fig. 1i).

To evaluate whether a preventive or a therapeutic setting contributes to the protection against DSSinduced colitis, we performed another experiment in which mice were supplemented with BL before (beBL) or during (duBL) the DSS treatment (Additional file 1: Fig. S1I). Notably, mice with beBL treatment exhibited improved body weight loss, DAI severity, and colon length shortening compared to mice with duBL treatment, producing effects similar to those in BL group (Additional file 1: Fig. S1 J-L). Similarly, histological analysis of colonic sections revealed that mice in CD + DSS and duBL + DSS groups exhibited serious injuries including crypt distortion, goblet cell loss and inflammatory cell infiltration in lamina propria and submucosa, which were markedly abrogated in beBL + DSS and BL + DSS group (Additional

file 1: Fig. S1M and N). Taken together, these results suggest that dietary supplementation of BL protects against DSS-induced colitis and gut microbiota dysbiosis, and these effects may largely depend on a preventive manner.

\section{BL enhances colonic motility and improves mucosal barrier function}

We speculated that the preventive impacts facilitated by BL on DSS-induced colitis might be explained by the possibility that dietary supplementation of BL could improve the intestinal functions before colitis induction. Investigation of intestinal morphology showed that BL-fed mice had a significant increase in crypt height and muscular layer width in the colon compared to control mice (Fig. 2a-c). Nevertheless, the altered morphology was not observed in the small intestine (Additional file 1: Fig. S2A). The coordinated contractions of muscular layer facilitate the motility of the intestine [29]. The increased colonic muscular layer width in BL-fed mice prompted us to speculate whether BL feeding could have an influence on intestinal motility. Compared with control mice, increased stool frequency and shortened gut transit time were observed in BL-fed mice (Fig. 2d and e). Moreover, scanning electron microscope images revealed that BL-fed mice displayed drastic alterations of ultra-morphology as indicated by obvious edges and corners on colonic mucosal surface (Fig. 2f), suggesting that BL might facilitate protective effects on colitis prevention through improving intestinal barrier functions. However, there was no significant differences in the expression of genes encoding for tight junction proteins such as zonula occludens 1 (ZO1), Occludin, Claudin2 and Claudin4 and antimicrobial peptides such as regenerating islet derived $3 \mathrm{~b}$ (Reg3b), Reg3g, Defensin4 and matrix metalloprotease 7 (Mmp7) (Additional file 1: Fig. S2B-I) between BL-fed and control mice. In addition, both groups of mice displayed comparable levels of macrophages and neutrophils infiltration, as evidenced by no major differences in the expression levels of F4/80 and Ly6G in colonic tissues (Additional file 1: Fig. S2J and K).

We next focused on the colonic mucus layer to better understand the impacts of $B L$ on mucosal barrier functions. As shown in Fig. $2 \mathrm{~g}$, alcian blue staining showed that the number of mucusproducing goblet cells was significantly increased in the colon of BL-fed mice compared to that of control mice. Of note, mucin granules in the goblet cells of BL-fed mice appeared to present enhanced apical exocytosis and 
secretion on the mucosal surface, which were not observed in control mice (Fig. 2g). Mucin2 (Muc2), the most abundant mucin protein that forms the mucus layer, is heavily glycosylated and secreted by goblet cells [10]. Immunohistochemical analysis revealed that there were also more Muc-2 positive goblet cells in the colon of BL-fed mice compared to that of control mice (Fig. 2h). Closer examination by transmission electron microscopy showed that the theca of colonic goblet cells in BL-fed mice contained large mucin granules (Fig. 2i), suggesting the enhanced biogenesis and accumulation of mucin protein in colonic goblet cells. These results suggest that $\mathrm{BL}$ improves intestinal functions possibly by promoting colonic motility and enhancing mucosal barriers.

\section{BL alters gut gene expression profile and activates PPARY signaling pathway}

To further delineate the mechanisms underlying the improvements of intestinal motility and mucosal barrier functions in BL-fed mice, we performed genome-wide transcriptional profiling of the whole colonic tissues for RNA-sequencing. There were significant differences in the transcriptomes between BL-fed and control mice (Fig. 3a). Based on significant false discovery rate $P$ value criteria (fold change $>1$ and $p<$ $0.05), 1735$ significantly altered genes were identified. Among them, 912 genes were upregulated and 823 genes were downregulated in the colon of BL-fed mice versus control mice. Kyoto Encyclopedia of Genes and Genomes (KEGG) pathway analysis of differentially expressed genes showed enrichment in a series of signaling pathways (Fig. 3b). Of note, PPAR signaling was the most significantly enriched functional pathway in BL-fed mice (Fig. 3b). Interestingly, 26 significantly changed genes that were enriched in PPAR signaling were mainly involved in nutrient metabolisms, such as lipid biosynthesis (Scd1), lipid degradation (Lpl, Adipoq), lipid storage (Plin1, Plin2, Plin4), lipid transport (Scp2, Slc27a1, Slc27a2), lipid binding (Fabp4, Fabp5, CD36, Ppara, Pparg), ß-oxidation (Cpt1a, Acs/1, Acs/3, Ehhadh) and cholesterol metabolism (Hmgcs1, Hmgcs2, Cyp27a1) (Fig. 3c). This was consistent with a previous study demonstrating that lipid metabolism is closely associated with improvements of mucosal barrier functions [30]. Furthermore, search tool for recurring instances of neighbouring genes (STRING) analysis was used to depict interaction networks of the significantly changed genes (Fig. 3d). Using quantitative real-time PCR, we confirmed the mRNA expression of key genes in PPAR signaling pathway and found that Pparg was the most significantly changed gene in BL-fed mice compared to control mice (Fig. 3e). Consequently, the increased protein expression of PPARy in colonic tissues of BL-fed mice was further confirmed by immunofluorescence imaging (Fig. 3f). Overall, these results suggest that BL feeding results in alteration of genes associated with nutrient metabolism and the activation of PPARY signaling may contribute to the impacts of BL on intestinal functions.

PPARy signaling pathway plays an essential role in the regulation of intestinal homeostasis [19]. To assess whether the PPARY signaling pathway was associated with improved intestinal functions, we treated mice with PPARY signaling antagonist GW9662 by daily oral gavage [19]. Consistent with the above impacts on intestinal morphology, $\mathrm{BL}$ feeding resulted in a higher crypt height and muscular layer width in the colon (Fig. 4a-C). Moreover, increased stool frequency and shortened gut transit time were also observed in BL-fed mice compared with control mice (Fig. 4d and e). However, when the PPARY 
signaling was suppressed by GW9662, the effects of BL on muscular layer width, stool frequency, intestinal transit time were abolished (Fig. 4c-e). PPARy signaling inhibition also abrogated the beneficial effects of $B L$ on mucosal barrier functions because BL failed to increase the number of mucusproducing goblet cells in GW9662-treated mice (Fig. 4f).

To determine whether PPARy signaling activation was required for the impacts of BL on colitis protection, we examined colitis symptoms in DSS-induced colitis model. As expected, BL-fed mice were protected from DSS-induced body weight loss, increased DAI scores and intestinal permeability, as well as colon shortening (Fig. 4g-j). However, PPARy signaling inhibition by GW9662 abolished the protective effects of BL on DSS-induced colitis (Fig. 4g-j). Additionally, the protective effects of BL on DSS-induced epithelial damage were also abrogated by GW9662 treatment (Fig. 4k and I). Collectively, these findings indicate that BL protects against DSS-induced colitis possibly through activating PPARy signaling pathway.

\section{BL promotes the enrichment of gut microbiota-derived purine metabolites}

Dietary nutrients have a great impact on the regulation of commensal microbial metabolites, which are considered pivotal mediators of host-microbiota interactions [16]. We hypothesized that the potential mechanisms by which BL influenced the intestinal functions is via the secondary metabolites generated during microbial fermentation in the gut. To assess metabolic alterations in response to $B L$ feeding, untargeted metabolomic profiles were generated on colonic contents and serum from BL-fed and control mice by high-resolution gas chromatography and mass spectrometry (GC-MS). As shown in Fig. 5a and b, the partial least squares-discrimination analysis (PLS-DA) model exhibited a significant separation of clusters between the two mouse groups. Heat map analysis showed that $B L$ feeding resulted in dramatic alteration of metabolites, with a total of 23 metabolites (16 up-regulated and 7 down-regulated) and 28 metabolites (14 up-regulated and 14 down-regulated) were significantly altered in mouse colonic contents and serum, respectively (Fig. $5 \mathrm{c}$ and d). The metabolomic map revealed the significant enriched pathway affected by BL feeding and most of these pathways were associated with energy and nutrient metabolisms such as glucose, lipid and amino acid metabolism (Fig. $5 \mathrm{e}$ and f). Notably, purine metabolism was the pathway that was significantly influenced by $B L$ in both the colonic contents and serum (Fig. 5e and f). Specifically, we found that inosine and guanosine, two major intermediate metabolites involved in purine metabolism, were the most significantly increased metabolites in response to $\mathrm{BL}$ feeding in both colonic contents and serum (Fig. $5 \mathrm{c}$ and d).

It has been known that inosine and guanosine are important foodstuff additives that are obtained at the industrial level mainly by microbial fermentation [31]. We further speculated that the gut microbiota might be associated with the enrichment of inosine and guanosine in BL-fed mice. To test this hypothesis, we treated BL-fed and control mice with broad-spectrum antibiotics to eliminate the gut micorbiota and further examined the levels of inosine and guanosine by targeted analysis. Comparing to the control mice, the BL-fed mice displayed significantly increased levels of inosine and guanosine in both colonic 
contents and serum (Fig. $5 g$ and $\mathrm{h}$ ). However, antibiotic treatment strongly decreased the levels of these two purine metabolites (Fig. $5 \mathrm{~g}$ and $\mathrm{h}$ ).

Based on the role of gut microbiota in modulating the enrichment of inosine and guanosine in BL-fed mice, we hypothesized that the gut microbiota might also play an important role in regulating the impacts of $\mathrm{BL}$ on mice intestinal functions. Accordingly, we performed untargeted metabolomics analysis to study differentially expressed small molecule metabolites in colonic tissues between BL-fed and control mice. As expected, principal components analysis (PCA) model and PLS-DA model exhibited a distinct clustering of metabolites between the two groups, indicating that $B L$ supplementation resulted in metabolic alterations in the colon (Additional file 1: Fig. S3A). Heat map analysis showed that 45 metabolites were significantly altered by BL feeding (Additional file 1: Fig. S3B). Among them, 14 metabolites were increased and 31 metabolites were decreased in BL-fed mice versus control mice (Additional file 1: Fig. S3B). Interestingly, the majority of metabolites that contribute highly to the metabolic distinction were relevant to energy pathways. Specifically, significant decrease of glycolysis substrates such as glucose, glucose-6-phosphate and fructose-6-phosphate, as well as significant increase of glycolysis product lactic acid were identified in BL-fed mice compared to control mice (Additional file 1: Fig. S3C-F). This was consistent with the above results of increased intestinal motility in BL-fed mice (Fig. 2), suggesting that BL promoted colonic motility possibly through enhanced glycolysis pathway. Notably, when the gut microbiota was suppressed by antibiotic treatment, the metabolic alterations caused by BL were abolished (Additional file 1: Fig. S3C-F). Thus, these data suggest that BL induces metabolic reprogramming in colonic tissues via a gut microbiota-dependent manner.

To directly assess the role of the gut microbiota in BL-mediated enrichment of purine metabolites, we measured the concentrations of inosine and guanosine after the incubation of BL with fresh mice feces for in vitro fermentation assay under strict anaerobic conditions (Additional file 1: Fig. S4A). Fermentation of $B L$ by the gut microbiota resulted in significantly increased levels of inosine and guanosine, which were not changed in vehicle batch cultures (Additional file 1: Fig. S4B and C).

To elucidate the mechanism responsible for the accumulation of inosine and guanosine, we performed $16 \mathrm{~S}$ rRNA gene sequencing to analyze the bacterial composition in batch cultures at $12 \mathrm{~h}$ and $24 \mathrm{~h}$. BL fermentation resulted in marked alterations of the bacterial communities, as revealed by a distinct clustering of microbiota composition for $12 \mathrm{~h}$ and $24 \mathrm{~h} \mathrm{BL}$ fermentation group compared to their respective vehicle control group (PERMANOVA, P < 0.05) (Additional file 1: Fig. S4D). Decreased adiversity was observed at each time point in $B L$ fermentation group, suggesting losses of particular bacterial taxa induced by BL fermentation (Additional file 1: Fig. S4E and F). Analyses of the microbiota at phylum level revealed significant increase in the relative abundance of Firmicutes and decrease in the relative abundance of Bacteroidetes in $B \mathrm{~L}$ fermentation group than in vehicle control group (Additional file 1: Fig. S4G and H). Notably, BL increased the relative abundance of Lactobacillus from $0.41 \% \pm 0.02-$ $25.89 \% \pm 7.46 \%$ and from $0.42 \% \pm 0.15-37.92 \% \pm 2.92 \%$ by $12 \mathrm{~h}$ and $24 \mathrm{~h}$ of fermentation, respectively (Additional file 1: Fig. S4I and J). Finally, Pearson correlation analyses showed that the abundance of Lactobacillus had positive relationships with the concentrations of inosine (Pearson correlation 
coefficients, $R=0.8883, P<0.0001$ ) and guanosine (Pearson correlation coefficients, $R=0.9266, P<0.01$ ) respectively in anaerobic cultures (Additional file 1: Fig. S4K and L). These data indicate that the enriched Lactobacillus may contribute to the enrichment of inosine and guanosine, which was consistent with the results of a previous study [32].

\section{Inosine activates PPARy signaling in human colon epithelial cells}

Given that microbial metabolites are in close contact with gut epithelium, we hypothesized that the metabolites could have a direct impact on intestinal epithelial cell functions. To examine such effects, we assessed the functional activity of inosine and guanosine in vitro. As shown in Fig. $6 \mathrm{a}$ and b, both metabolites with different concentrations $(0.5,1,2$ and $4 \mathrm{mM})$ showed no discernible toxicity in human colon epithelial HT-29 cells (Fig. 6a and b). Next, a luciferase-based system was used to detect the bioactivity of inosine and guanosine on PPARY signaling. Interestingly, the PPARy-luciferase activity was significantly increased in cells treated with inosine or in cells treated with rosiglitazone, but not in guanosine-treated cells (Fig. $6 \mathrm{c}$ and d), suggesting that inosine but not guanosine might be the metabolite responsible for BL-induced PPARY signaling activation. We also observed significantly increased expression of key genes in PPARy signaling pathway, such as lipid degradation gene $L p l$, lipid biosynthesis gene Scd1, lipid binding genes CD36, Fabp4, lipid transport gene S/c27a 1 and $\beta$-oxidation gene Cpt1a after inosine treatment in both HT29 cells and another colon epithelial cell line, Caco2 (Fig. 6e and $f$ ). The elevated protein level of PPARy was further confirmed by western blot and immunofluorescence imaging (Fig. 6g-i). To investigate whether PPARY was required for inosine-mediated induction of mucin protein, PPARy expression was suppressed using siRNA and the expression of Muc2 was examined. Interestingly, the level of Muc2 was significantly increased by inosine treatment. However, inosine failed to induce Muc2 in PPARy knockdown cells (Fig. 6j and k). Because inosine has been reported as a ligand for adenosine $2 A$ receptor $\left(A_{2 A} R\right)$ [21], we tested whether inosine mediated PPARY signaling activation through $A_{2 A} R$. As shown in Fig. $6 \mathrm{l}$ and $\mathrm{m}$, inosine failed to induce PPARY and Muc2 in $A_{2 A} R$ knockdown cells. These results suggest that inosine induces the expression of mucin protein at least in part through the $A_{2 A} R / P P A R y$ pathway.

Inosine improves intestinal functions and protects against colitis via $A_{2 A} R / P P A R Y$

We next determined the in vivo role of $A_{2 A} R / P P A R y$ as a mediator of the beneficial effects induced by inosine (Fig. 7a). Consistent with the above in vitro data, inosine treatment resulted in significantly increased expression of PPARY and its target genes ( $L p l, S c d 1, C D 36$ and Fabp4) in colonic tissues (Fig. 7b and C). Meanwhile, treatment of inosine partially phenocopied the effects of BL on intestinal morphology, as evidenced by significantly increased muscular layer width in colon of inosine-treated mice (Fig. 7d and e). Inosine also enhanced gut motility via increasing the stool frequency and decreasing the gut transit time (Fig. $7 f$ and $g$ ). In addition, our results revealed that inosine treatment improved mucosal barrier functions by increasing the number of mucus-producing goblet cells (Fig. 7h). However, inosine failed to induce these effects in mice treated with GW9662 or $A_{2 A} R$-specific antagonist SCH58261 
(Fig. 7e-h). We then examined whether inosine contributed to the protective effects of BL on DSS-induced colitis. As shown in Fig. 7i-k, inosine reduced DSS-induced body weight loss, attenuated DAl scores and colon shortening. We also observed that inosine effectively enhanced barrier function as evident by improved intestinal permeability and histological damage in DSS-treated mice (Fig. 7l-n). Nevertheless, the beneficial effects of inosine on colitis were reversed when mice were treated with GW9662 or SCH58261 (Fig. 7i-n). Out data thus reveal that inosine exerts the protective effects against colitis partly through $A_{2 A} R / P P A R y$ signaling.

\section{Discussion}

Ulcerative colitis is emerging as a health-threatening disease in the world [4]. Conventional drug-based therapies have led to substantial economic costs and certain side effects [33]. Hence, there remains an urgent need for the development of effective and safe treatment agents. BL, an herbal-based dietary supplement in Traditional Chinese Medicine, is an attractive candidate due to its potent health benefits on intestinal functions. In this study, we found that dietary supplementation of BL protected against DSSinduced colitis and gut microbiota dysbiosis. Moreover, we identified for the first time that inosine, a gut microbial purine metabolite, contributed to the protective effects of BL against DSS-induced colitis by improving $A_{2 A} R / P P A R y$-dependent mucosal barrier functions (Additional file 1: Fig. S5). Thus, our results suggest that the microbiota-inosine- $A_{2 A} R / P P A R y$ axis could have tremendous potential for preventing and treating intestinal inflammatory disorders, such as colitis.

The intestinal tract is colonized by a large and diverse microbial community, which plays a critical role in the maintenance of intestinal homeostasis $[34,35,36]$. A growing body of evidences has suggested that gut microbiota dysbiosis appears to be associated with the pathogenesis of ulcerative colitis [37]. For instance, compared with healthy subjects, the gut microbiota of inflammatory bowel disease patients exhibited a reduction in microbial diversity $[38,39]$. In line with these findings, our $16 \mathrm{~S}$ rRNA gene sequencing data also showed that DSS treatment caused a reduced gut microbial richness and diversity in mice, which was effectively rescued by BL supplementation. The dysbiosis-associated reduction in gut microbial diversity may be due to a shift in the balance between commensal and potentially pathogenic microorganisms. Indeed, it has been reported that a healthy gut microbiota composition is dominated by anaerobic Firmicutes and Bacteroidetes, while microbiota dysbiosis is featured by the expansion of facultative anaerobic pathogens belonging to the Enterobacteriaceae family $[28,40]$. In the present study, we found that BL significantly suppressed the increased abundance of Enterobacteriaceae in DSS-treated mice. The mechanism by which BL inhibited DSS-induced gut microbiota dysbiosis may partially result from its ability to activate PPARY signaling in the colon, because a recent study reports that PPARY activation can limit gut lumen oxygen level by driving $\beta$-oxidation in colonic epithelial cells, thereby inhibiting the expansion of pathogenic Enterobacteriaceae [19].

Apart from the gut microbiota, other crucial factors that are also involved in the maintenance of intestinal homeostasis include intestinal motility and barriers, because dysfunction of intestinal motility and mucosal barriers are common symptoms frequently presented in patients with inflammatory bowel 
disease $[41,42]$. In this study, we found that the protection of BL against DSS-induced colitis was through a preventive manner which might largely attribute to its beneficial effects on intestinal functions. The increased crypt height and muscular layer width observed in BL-treated mice indicated that dietary supplementation of $\mathrm{BL}$ induced enhanced intestinal motility before colitis induction, which was also confirmed by the increased stool frequency and shortened gut transit time. It was noted that the BLinduced morphological alterations were specific to the large intestine where high density of microorganisms is present, suggesting the role of gut microbiota in the regulation of these effects. Additionally, metabolomics analysis showed that BL caused metabolic reprogramming in mice colon characterized by the promotion of glycolysis process, which may partially explain the enhanced intestinal motility. However, antibiotic treatment abrogated the metabolic alterations in BL-fed mice, suggesting that BL might promote intestinal motility in a gut microbiota-dependent manner.

We next applied RNA-sequencing to investigate the underlying mechanisms of BL on intestinal functions. Notably, we noted significant increase in the expression of genes involved in biosynthesis, degradation, storage, transport and binding of lipid. A previous study has revealed an essential role of de novo lipogenesis enzyme fatty acid synthase in maintaining intestinal barrier function through modulating the palmitoylation of Muc2 [30]. Thus, the increased expression of genes associated with lipid metabolism may explain the improvements of mucosal barrier function in BL-fed mice. Further functional analysis showed that PPAR signaling was the most significantly enriched functional pathway in response to BL feeding. PPARy is an essential nuclear receptor that mainly expressed in adipose tissue and involved in the regulation of insulin resistance by controlling the expression of a large number of regulatory genes in lipid metabolism. Notably, PPARy is also highly expressed in the colon, while its expression is impaired in patients with ulcerative colitis [43]. The mechanisms by which PPARY signaling modulate the intestinal homeostasis have been reported to be associated with the modulation of colon epithelium cell differentiation [44], adaptive immune effector T cell function [45], and innate immune antimicrobial response [46]. Moreover, PPARy signaling also plays an important role in regulating the impacts of high fat diet on the mucosal immune defenses in small intestine [47]. In line with these findings, our data showed that inhibition of PPARY signaling by GW9662 abrogated the protective effects of BL on colonic motility and mucosal barrier, as well as the protection against DSS-induced colitis.

Given the pivotal role in the modulation of intestinal homeostasis, PPARy has been recognized as an important target for the treatment of inflammatory bowel disease. For instance, troglitazone and rosiglitazone are the two PPARy synthetic ligands that can dramatically reduce disease severity in experimental colitis models and in patients with ulcerative colitis $[48,49,50]$. Moreover, PPARy is also an important target of 5-aminosalicylic acid, one of the oldest anti-inflammatory agents used for the treatment of inflammatory bowel disease [51]. Interestingly, many natural ligands that present in food such as conjugated linoleic acid have been reported to efficiently prevent the development of colitis through PPARy signaling activation [52]. Notably, conjugated linoleic acid is a metabolite that can be generated by gut bacteria Lactobacillus, indicating the involvement of gut microbiota in the regulation of PPARy signaling [53]. Correspondingly, another study has demonstrated that the microbial fermentation product butyrate induces PPARY signaling activation in vitro and in vivo [54]. To search for the potential 
agonists responsible for BL-induced PPARy signaling activation, an untargeted metabolomics analysis was performed. We found that supplementation of BL resulted in the enrichment of purine nucleotides, inosine and guanosine in both colonic contents and serum. We examined their activities by using in vitro functional analysis. Our results revealed that the activity and expression of PPARy could be induced by the treatment of inosine but not guanosine in human colon epithelial cells, suggesting that inosine may be a potent agonist for PPARY signaling activation. Furthermore, exogenous treatment with inosine activated colonic PPARY signaling and protected against DSS-induced colitis through improving mucosal barrier functions. Notably, no obvious toxicity and side effects were observed in our in vitro and in vivo experiments, suggesting that inosine has the potential to be developed as a promising agent for the treatment of colitis. Nevertheless, further study will be required to determine the efficacy and safety of inosine in ulcerative colitis patients.

It has been known that inosine and guanosine are important foodstuff additives that have synergistic effects with monosodium glutamate to increase the intensity of the "umami" taste [55]. At the industrial level, microbial fermentation technology is the most efficient method for their production. By using genetic engineering, several bacterial strains such as Bacillus subtilis [56], Corynebacterium ammoniagenes [57] and Escherichia coli [58] have been applied to increase the production of these purine nucleotides. It should be noted that the production of inosine and guanosine can also be obtained by metabolic engineering of a filamentous fungus Ashbya gossypii [31], suggesting the potential use of both eukaryotic and prokaryotic microorganisms in industrial nucleoside production. In the present study, antibiotic treatment and anaerobic fermentation experiments confirm our hypothesis that the gut microbiota plays an indispensable role in promoting the enrichment of purine metabolites, which was consistent with the results of the recent study [22]. Interestingly, the 16S rRNA gene sequencing of bacterial composition revealed an increased abundance of Lactobacillus during BL fermentation, suggesting the role of Lactobacillus in regulating the enrichment of these purine metabolites. This is supported by a recent study demonstrating that Lactobacillus reuteri can inhibit regulatory $T$ cell deficiency-induced autoimmunity via inosine- $A_{2 A} R$ signaling [32]. In the present study, the inhibition of $A_{2 A} R$ by antagonist ligand $S C H 58261$ blocked the effects of inosine on PPARY signaling activation and mucosal barrier improvements, suggesting that $A_{2 A} R$ may be a novel target for the treatment of intestinal disorders [59].

\section{Conclusions}

In the present study, we demonstrate that dietary supplementation of BL can protect against DSS-induced colitis and microbiota dysbiosis by promoting intestinal motility and improving mucosal barrier functions. In addition, BL promotes the enrichment of microbiota-derived purine metabolite inosine, which induces $A_{2 A} R / P P A R y$ signaling activation and contributes to the impacts of $B L$ on the improvements of intestinal functions. Our results highlight that $\mathrm{BL}$ and inosine might be used as efficacious supplements in individuals with intestinal dysfunction. 


\section{Methods}

\section{Preparation of BL powder}

BL powder used in this study was crude powder without any extraction process and was supplied by Hebei Biotechnology Co., Ltd. (Jiaxing, China). Briefly, fresh leaves of Hordeum vulgare L. (cultivated in Hangzhou, China) were washed with water, cut into pieces and dried in a freeze dryer OE-950 (Labor, MIM, Budapest, Hungary) at $-60^{\circ} \mathrm{C}$ for $24 \mathrm{~h}$. The dried BL was pulverized with a blender (KA-2610, Jworld Tech, Korea) for $1 \mathrm{~min}$, screened through a 300 mesh sieve and stored at $-20^{\circ} \mathrm{C}$ until use. The proximate analysis of the BL powder was performed by Pony Testing International Group (Beijing, China) (Additional file 2: Table S1).

\section{Animal experiments}

Eight-week-old female C57BI/6J mice were obtained from Vital River Laboratory Animal Technology (Beijing, China) and maintained under specific-pathogen-free (SPF) facility with a 12-h light and dark cycle. All mice were adapted to the laboratory conditions with ad libitum access to food and water for at least one week. Animal experiments were conducted in accordance with the Guidelines for Animal Experimentation of Peking University Health Science Center (Beijing, China), and the protocols were approved by the Animal Ethics Committee of this institution. Mice were randomly distributed and fed with either a standard chow diet (CD) or an isocaloric diet where BL was supplemented at a ratio of $2.5 \%$, a dosage translated from a previous human intervention study [24]. The composition of two diets was listed in Additional file 2: Table S2 and prepared by Hfk Biotech Co, Ltd (Beijing, China).

For the treatment with PPARY antagonist GW9662 or $\mathrm{A}_{2 \mathrm{~A}} \mathrm{R}$ antagonist $\mathrm{SCH} 58261$, mice were treated with $3 \mathrm{mg} / \mathrm{kg} /$ day of GW9662 intragastrically [19] or treated with $2 \mathrm{mg} / \mathrm{kg} /$ day of SCH58261 intraperitoneally [32].

For antibiotic experiment, a combination of neomycin $(100 \mathrm{mg} / \mathrm{l})$, streptomycin $(50 \mathrm{mg} / \mathrm{l})$, penicillin (100 $\mathrm{mg} / \mathrm{l})$, vancomycin $(50 \mathrm{mg} / \mathrm{l})$ and metronidazole $(100 \mathrm{mg} / \mathrm{l})$ were administered in the drinking water.

For the treatment with inosine, mice were treated intragastrically with $800 \mathrm{mg} / \mathrm{kg} /$ day of inosine (SigmaAldrich) dissolved in PBS at the concentration of $40 \mathrm{mg} / \mathrm{ml}$ [32].

\section{Dextran sodium sulfate (DSS)-induced colitis}

Colitis was induced by administering 2.5\% DSS (molecular weight 36,000-50,000 kDa; MP Biomedicals) dissolved in drinking water for 7 days. The mice were weighed daily and monitored for signs of stool consistency and rectal bleeding. Evaluation of disease activity index was performed by combining the parameters of weight loss, stool consistency and rectal bleeding as described previously [60]. The disease activity index was the mean of the total score of the three parameters. Mice were sacrificed on day 7 and colon length was measured.

\section{Assessment of intestinal permeability}

Page 13/36 
Briefly, fluorescein isothiocyanate (FITC)-dextran (4 kDa; Sigma) was dissolved in PBS at a concentration of $100 \mathrm{mg} / \mathrm{ml}$. After mice were fasted for $4 \mathrm{~h}$ and orally administrated with FITC-dextran $(60 \mathrm{mg} / 100 \mathrm{gm}$ body weight). Blood was collected following another $4 \mathrm{~h}$ and was centrifuged at 1,000 rpm for $20 \mathrm{~min}$.

Serum was collected and fluorescence was quantified at an excitation wavelength of $485 \mathrm{~nm}$ and $535 \mathrm{~nm}$ emission wavelength.

\section{S rRNA gene sequencing and analysis}

Total DNA was extracted from colonic contents or fecal culture samples using a QIAamp-DNA Stool Mini Kit (Qiagen, Hilden, Germany). The integrity of the extracted DNA was examined by electrophoresis in 1\% $(\mathrm{wt} / \mathrm{vol})$ agarose gels. Based on the quantity and the quality of the DNA extracted, samples were selected to perform the consequent sequencing.

DNA samples were used as the template for PCR amplification of the V3-V4 region of bacterial 16S rRNA genes. PCR amplification was performed on ABI GeneAmp ${ }^{\circledR} 9700$ PCR System (AppliedBiosystems, Foster City, CA, USA) and the PCR amplification products were quantified with a QuantiFluor ${ }^{\text {TM_ST }}$ Handheld Fluorometer with UV/Blue Channels (Promega Corporation, Madison, WI, USA)

Sequencing of the PCR amplification products was performed on an Illumina Miseq platform at Majorbio Bio-Pharm Technology Co., Ltd. (Shanghai, China). Briefly, the 16S rRNA gene sequencing data was filtered and trimmed, and further classified into operational taxonomic units (OTUs) within a 0.03 difference (equivalent to $97 \%$ similarity). A representative set of sequences was then generated and was assigned taxonomy using the SILVA database (Release115 http://www.arb-silva.de). Analyses for rarefaction curves, and calculation of richness estimators and diversity indices were performed using the MOTHUR program. Taxonomic community structure and phylogeny were analyzed through visualization of the data sets of the microbial diversities and abundances of different samples.

\section{Inflammatory cytokine analysis}

Cytokine levels in serum and colonic tissues were detected by enzyme-linked immunosorbent assay (ELISA) kit (Nanjing Jiancheng Bioengineering Institute Co., Ltd. Nanjing, China) according to the manufacturer's instructions.

\section{Histological evaluation}

Collected colon samples were fixed in $4 \%$ paraformaldehyde and embedded in paraffin. Paraffinembedded colonic tissues were sectioned ( $4 \mu \mathrm{m}$ in thicknesses) and were subjected to hematoxylin and eosin (HE) and alcian blue (AB) staining by using commercial kits (Beijing Solarbio Technology Co., Ltd. Beijing, China) according to the manufacturer's instructions. The crypt height and muscular layer width in the large intestine were measured using Image J (Media Cybernetics, Inc., Rockville, MD, USA). Mucusproducing goblet cells were observed and counted under light microscope (Leica DM500). Six microscopic fields of every section of the testes were randomly selected. 
Histological pathology was scored according to a previously established scoring system, as follows: crypt damage ( $0-4$ scale), severity of inflammation ( $0-3$ scale) and depth of injury ( $0-3$ scale) [61].

\section{Intestinal motility assessment}

The intestinal motility was determined through evaluating the intestinal transit time and defecation frequency. After mice were fasted for $4 \mathrm{~h}$, individual mouse was gavaged with the $10 \mu \mathrm{L} / \mathrm{g}$ of $6 \%$ carmine solution (in $0.5 \%$ methylcellulose). The time from gavage to initial appearance of carmine in the feces was recorded as the gut transit time for a given mouse.

Defecation frequency was recorded by separating mice into a sterile cage and the cumulative fecal pellets within 60 min were recorded as the corresponding defecation number for a given mouse.

\section{Scanning electron microscopy}

Colonic tissues were fixed with 2.5\% PBS-diluted glutaraldehyde. After being rinsed three times in PBS, samples were post-fixed in $1 \%$ osmium tetroxide for $1 \mathrm{~h}$, dehydrated in alcohol, and then critical-pointdried using $\mathrm{CO}_{2}$. The samples were coated with gold and observed in a Hitachi S-3400 scanning electron microscope (Hitachi, Japan).

\section{Transmission electron microscopy}

Colonic tissues were fixed with $2.5 \%$ glutaraldehyde. PBS was used to remove excess fixative. Samples were then fixed with $1 \%$ osmium tetroxide at $4^{\circ} \mathrm{C}$ for $2 \mathrm{~h}$, dehydrated in acetone. Samples were finally infiltrated with 1:1 propylene oxide and EPON resin for $1 \mathrm{~h}$ followed by overnight infiltration in $100 \%$ EPON's resin. The tissues were embedded in flat molds in $100 \%$ EPON for $36 \mathrm{~h}$ at $60^{\circ} \mathrm{C}$. Ultra-thin sections of $70 \mathrm{~nm}$ were stained with uranyl acetate and lead citrate (10 min each) and viewed under $\mathrm{H}-7650$ transmission electron microscope (Hitachi, Japan).

\section{Immunohistochemistry and immunofluorescence}

Following deparaffinization and rehydration, colonic sections were blocked with $5 \%$ bovine serum albumin for $30 \mathrm{~min}$ at room temperature and then washed with PBS. Tissue sections were incubated with primary antibody Muc-2 (Santa Cruz, sc-515032), Ly6G (Abcam, ab25377) and F4/80 (Abcam, ab6640) overnight at $4^{\circ} \mathrm{C}$. Slides were washed three times in PBS before applying peroxidase-conjugated secondary antibody (Invitrogen) for $2 \mathrm{~h}$ at room temperature.

For immunofluorescence staining, paraffin-embedded colonic sections or cells were incubated with primary antibody PPARy (Abcam, ab59256) at $4^{\circ} \mathrm{C}$ overnight. Next day, Alexa Fluor488 donkey antirabbit secondary antibody (Invitrogen) was used.

\section{Quantitative real-time PCR (qPCR)}

Total RNA of colonic tissues was isolated by using TRIZOL reagent (Invitrogen, USA). The quantity and purity of RNA was assessed by absorbance at $260 \mathrm{~nm}$ and $280 \mathrm{~nm}$. Reverse transcription of $10 \mu \mathrm{l} \mathrm{RNA}$ was performed using PrimeScript RT Master Kit (Takara, Japan). Gene expression was measured in a 
MyiQ single color Real-Time PCR detection system (Bio-Rad) with SYBR Real time PCR Kit (Takara, Japan). GAPDH was used as endogenous control. Average Ct values from triplicate analyses were normalized from average $\mathrm{Ct}$ values of GAPDH. The primer sequences are described and synthesized by Sunbiotech Co. (Beijing, China) (Additional file 2: Table S3).

\section{RNA sequencing}

Colon tissues were collected and the total RNA was extracted by using TRIZOL reagent (Invitrogen, USA). RNA quality was evaluated by electrophoresis using an Agilent 2100 Bioanalyzer (Agilent Technologies, San Diego, CA, USA). Samples with RNA integrity numbers (RINs) $>9.4$ and with 260/280 $\mathrm{nm}$ absorbance ratios from 1.9 to 2.1 were used for construction of RNA-Seq libraries. Libraries were constructed using the TruSeq ${ }^{\text {TM }}$ RNA Sample Prep kit (Illumina, San Diego, CA, USA) according to the manufacturer's instructions.

Sequencing of the Libraries was performed on an Illumina HiSeq2000 instrument by Shanghai Majorbio Biopharm Biotechnology (Shanghai, China), and individually assessed for quality using FastQC. Analysis of differential expression was carried out using DESeq2 [62]. Statistical significance was assessed using a negative binomial Wald test, then corrected for multiple hypothesis testing with the Benjamini-Hochberg method. Functional enrichment cluster analysis was performed for Kyoto Encyclopedia of Genes and Genomes (KEGG) pathway enrichment analysis.

\section{Non-targeted metabolomics}

Gas chromatography-mass spectrometry (GC-MS)-based metabolomics was performed by ProfLeader Biotech (Shanghai, China). Briefly, serum samples were separated from the blood by centrifugation and stored at $-80^{\circ} \mathrm{C}$. Colonic contents or colon tissue samples mixed with water were vortexed prior to centrifugation. The supernatant was transferred to a GC vial containing internal standards. The mixture was dried under gentle nitrogen stream and then added with methoxyamine hydrochloride in pyridine. The resultant mixture was vortexed vigorously and incubated at $37^{\circ} \mathrm{C}$ for $90 \mathrm{~min}$. Derivatization was performed by adding BSTFA (with 1\% TMCS) into the mixture. The derivatized samples were analyzed by an Agilent 7890A gas chromatography system coupled to an Agilent $5975 \mathrm{C}$ inert MSD system (Agilent Technologies, CA, USA). A HP-5MS fused-silica capillary column was utilized to separate the derivatives. Helium was used as a carrier gas at a constant flow rate through the column. The samples were analyzed in a random sequence.

The acquired GC/MS data were imported to SIMCA Statistical Analysis (version 13.0, Umetrics AB, Umeå, Sweden), where multivariate statistical analysis including principal component analysis (PCA) and partial least-squares discriminant analysis (PLS-DA) were performed. The differential metabolites were determined by the combination of the Variable importance in the projection (VIP) value ( $>1)$ of PLS-DA model and the $P$ values $(<0.05)$ from two-tailed Student's $t$ test on the normalized peak intensities. Fold change was calculated as a binary logarithm of the average normalized peak area ratio between the two groups. The structural identification of differential metabolites was performed using AMDIS software, where the purified mass spectra were automatically matched with an in-house standard library including 
retention time and mass spectra, Agilent Fiehn GC/MS Metabolomics RTL library and Golm Metabolome Database, respectively.

\section{Quantitative analysis of purine metabolites}

Serum, colonic contents or fecal culture samples were mixed with distilled water containing internal standard (4-aminosalicylic acid). The mixture was extracted by ethyl acetate. Following centrifugation, the top layer was transferred and evaporated to dryness under nitrogen stream. The dry residue was reconstituted in acetonitrile and BSTFA (with 1\% TMCS), and derivatized to perform GC-MS analysis. A mixed standard solution containing inosine and guanosine stock standard solutions was prepared at a concentration of $100 \mu \mathrm{g} / \mathrm{mL}$ for quantitation.

\section{In vitro anaerobic culturing}

Fresh mice feces $(0.5 \mathrm{~g})$ from 8-week-old male C57BI/6 mice were resuspended in $10 \mathrm{ml}$ sterile PBS and co-cultured with BL powder with a final concentration of $10 \mathrm{mg} / \mathrm{ml}$ under anaerobic conditions $\left(10 \% \mathrm{H}_{2}\right.$, $10 \% \mathrm{CO}_{2}, 80 \% \mathrm{~N}_{2}$ ). After incubation for 12 and $24 \mathrm{~h}$, cultured samples were collected and used for metabolites detection and bacterial composition analysis.

\section{Cell culture and treatment}

Human colon epithelial carcinoma cell lines HT29 and Caco2 were purchased from the American Type Culture Collection (ATCC). Cells were maintained in Dulbecco's modified Eagle's medium (DMEM) supplemented with 10\% fetal bovine serum (FBS) and 1\% penicillin/streptomycin in a humidified incubator $\left(5 \% \mathrm{CO}_{2}, 95 \%\right.$ air, $\left.37^{\circ} \mathrm{C}\right)$. Cells were grown until 80 to $90 \%$ confluence and treated for the indicated time periods with inosine (Sigma-Aldrich) and guanosine (Sigma-Aldrich) from a stock in PBS to a final concentration of $0.5,1,2$ and $4 \mathrm{mM}$. Cell viability was evaluated with the Cell Counting Kit (CCK)-8 cell viability assay kit from GenMed Inc. (Shanghai, China) according to the manufacture's instruction.

\section{Luciferase reporter assay}

PPARy-luciferase plasmid vector was purchased from RiboBio Co., Ltd (Guangzhou, China). HT29 cells were transiently transfected using lipofectamine 3000 reagent (ThermoFisher Scientific). Briefly, $1 \times 10^{5}$ cells were seeded on 6-well plates and grown for $24 \mathrm{~h}$. The transfection complex containing $1 \mu \mathrm{g}$ of plasmid DNA and transfection reagent was added to each well in absence of FBS. After $6 \mathrm{~h}$, medium containing $10 \%$ FBS was added and cells were treated with inosine and guanosine $(0.5,1,2$ and $4 \mathrm{mM})$ for $24 \mathrm{~h}$. The luciferase activity was measured with Luciferase Assay System (Promega) using a luminometer (Perkin Elmer, Covina, CA).

\section{Western blot analysis}

Cells were lysed with lysis buffer with $1 \%$ protease inhibitor cocktail to harvest total cellular protein. The concentration of extracted protein was quantified by bicinchoninic acid (BCA) protein assay kit (Beyotime). An equal amount of protein sample was loaded on sodium dodecyl sulphate-polyacrylamide 
gel electrophoresis gel and then transferred to a polyvinylidene difluoride membrane (Millipore, Billerica, MA, USA). After the membrane was blocked with skimmed milk, it was incubated with primary antibodies against Muc2 (Santa Cruz, sc-515032), PPARy (Abcam, ab59256), $A_{2 A} R$ (Abcam, ab3461) and GAPDH (Abcam, ab8245). Next day, secondary antibodies conjugated with Horseradish peroxidase were probed for 2-3 h. Protein signals were detected with enhanced ECL chemiluminescence reagent based on the manufacturer's instructions.

\section{Small interfering RNA (siRNA)}

The siRNA against PPARY or $A_{2 A} R$ was purchased from GenePharma (Shanghai, China). For knockdown experiments, $1 \times 10^{5} \mathrm{HT} 29$ and Caco2 cells were plated in 6 well plates and grown for $24 \mathrm{~h}$. The PPARY, $A_{2 A} R$ or control-siRNA was transfected into cells using lipofectamine 3000 reagent (ThermoFisher Scientific). After $24 \mathrm{~h}$ of transfections, cells were induced with inosine $(0,2$ and $4 \mathrm{mM})$ for $24 \mathrm{~h}$. And then cells were lysed using RIPA buffer and the protein expression of $A_{2 A} R$, PPARy or Muc2 was detected by western blot.

\section{Statistical analysis}

Statistical analysis was performed using Prism 6.0 (GraphPad Software, CA). Data are presented as means \pm SEM. Significant differences between the two groups were evaluated by two-tailed unpaired Student's $t$ test. Significant differences more than two groups were evaluated by one-way or two-way analysis of variance (ANOVA) followed by Tukey's multiple comparison's test.

When analyzing gut microbiota sequencing data, two-tailed Wilcoxon rank-sum test by R Project were performed. When analyzing the differences of abundance distributions among metabolites, MannWhitney $\mathrm{U}$ test with Benjamini-Hochberg false discovery rate correction were performed. The differences were considered to be significant at $p<0.05$.

\section{Declarations}

\section{Ethics approval and consent to participate}

All experimental procedures were conducted in compliance with the institutional guidelines for the care and use of laboratory animals in China and approved by the Animal Ethical Council of Peking University Health Science Center. Animal welfare and experimental protocols were strictly in accordance with the guidelines for the care and use of laboratory animals.

\section{Consent for publication}

Not applicable.

\section{Availability of data and material}


The sequences generated for in this study are stored in the National Center for Biotechnology Information (NCBI) and the project numbers are PRJNA511491 and PRJNA511549. Additionally, all other data is contained within the main manuscript and supplemental files.

\section{Competing interests}

The authors declare that they have no competing interests.

\section{Funding}

This work was supported by National Natural Science Foundation of China [grant number 32001677], Beijing Municipal Science and Technology Project Fund [grant number D161100005416001], China Postdoctoral Science Foundation [grant number 2020M680256] and the Chinese Universities Scientific Fund [grant number 2016SP003].

\section{Authors' contributions}

$\mathrm{DL}, \mathrm{JJ}, \mathrm{XH}$ and $\mathrm{FC}$ conceived the idea and designed this study. DL, YF and MT performed the experiments. DL and FC wrote the manuscript, prepared the figures and was responsible for data compilation and integration. All authors contributed to discuss the results and to research directions. All authors approved the manuscript.

\section{Acknowledgements}

The authors would like to thank Dr. Zhanjun Hou (Department of Oncology, Wayne State University) and Dr. Li-Shu Wang (Medical College of Wisconsin) for critical reading of this manuscript.

\section{Authors' information}

${ }^{1}$ College of Food Science and Nutritional Engineering, National Engineering Research Center for Fruit and Vegetable Processing, Key Laboratory of Fruits and Vegetables Processing, Ministry of Agriculture; Engineering Research Centre for Fruits and Vegetables Processing, Ministry of Education, China Agricultural University, Beijing 100083, China. ${ }^{2}$ Department of Anatomy, Histology and Embryology, Health Science Center, Peking University, Beijing, China.

\section{References}

1. Xavier RJ, Podolsky DK. Unravelling the pathogenesis of inflammatory bowel disease. Nature. 2007;448(7152):427-34.

2. Yadav V, Varum F, Bravo R, Furrer E, Bojic D, Basit AW. Inflammatory bowel disease: exploring gut pathophysiology for novel therapeutic targets. Transl Res. 2016;176:38-68.

3. Kaplan GG. The global burden of IBD: from 2015 to 2025. Nat Rev Gastroenterol Hepatol. 2015;12(12):720-7. 
4. Ng SC, Shi HY, Hamidi N, Underwood FE, Tang W, Benchimol El, Panaccione R, Ghosh S, Wu JCY, Chan FKL, Sung JJY, Kaplan GG. Worldwide incidence and prevalence of inflammatory bowel disease in the 21 st century: a systematic review of population-based studies. Lancet. 2018;390(10114):2769-78.

5. Ordás I, Eckmann L, Talamini M, Baumgart DC, Sandborn WJ. Ulcerative colitis. Lancet. 2012;380(9853):1606-19.

6. Bouguen G, Langlois A, Djouina M, Branche J, Koriche D, Dewaeles E, Mongy A, Auwerx J, Colombel JF, Desreumaux P, Dubuquoy L, Bertin B. Intestinal steroidogenesis controls PPARy expression in the colon and is impaired during ulcerative colitis. Gut. 2015;64(6):901-10.

7. Decara J, Rivera P, López-Gambero AJ, Serrano A, Pavón FJ, Baixeras E, Rodríguez de Fonseca F, Suárez J. Peroxisome proliferator-activated receptors: experimental targeting for the treatment of inflammatory bowel diseases. Front Pharmacol. 2020;11:730.

8. Danese S. New therapies for inflammatory bowel disease: from the bench to the bedside. Gut. 2012;61:918-32.

9. David LA, Maurice CF, Carmody RN, Gootenberg DB, Button JE, Wolfe BE, Ling AV, Devlin AS, Varma Y, Fischbach MA, Biddinger SB, Dutton RJ, Turnbaugh PJ. Diet rapidly and reproducibly alters the human gut microbiome. Nature. 2014;505(7484):559-63.

10. Tytgat KM, Büller HA, Opdam FJ, Kim YS, Einerhand AW, Dekker J. Biosynthesis of human colonic mucin: Muc2 is the prominent secretory mucin. Gastroenterology. 1994;107:1352-63.

11. Jakobsson HE, Rodríguez-Piñeiro AM, Schütte A, Ermund A, Boysen P, Bemark M, Sommer F, Bäckhed F, Hansson GC, Johansson ME. The composition of the gut microbiota shapes the colon mucus barrier. EMBO Rep. 2015;16(2):164-77.

12. Johansson ME, Jakobsson HE, Holmén-Larsson J, Schütte A, Ermund A, Rodríguez-Piñeiro AM, Arike L, Wising C, Svensson F, Bäckhed F, Hansson GC. Normalization of Host Intestinal Mucus Layers Requires Long-Term Microbial Colonization. Cell Host Microbe. 2015;18(5):582-92.

13. De Palma G, Lynch MD, Lu J, Dang VT, Deng Y, Jury J, Umeh G, Miranda PM, Pigrau Pastor M, Sidani S, Pinto-Sanchez MI, Philip V, McLean PG, Hagelsieb MG, Surette MG, Bergonzelli GE, Verdu EF, BritzMcKibbin P, Neufeld JD, Collins SM, Bercik P. Transplantation of fecal microbiota from patients with irritable bowel syndrome alters gut function and behavior in recipient mice. Sci Transl Med. 2017;9(379):eaaf6397.

14. Manichanh C, Borruel N, Casellas F, Guarner F. The gut microbiota in IBD. Nat Rev Gastroenterol Hepatol. 2012;9(10):599-608.

15. Li D, Wang P, Wang P, Hu X, Chen F. The gut microbiota: A treasure for human health. Biotechnol Adv. 2016;34(7):1210-24.

16. Li D, Wang P, Wang P, Hu X, Chen F. Targeting the gut microbiota by dietary nutrients: A new avenue for human health. Crit Rev Food Sci Nutr. 2019;59(2):181-95.

17. Yano JM, Yu K, Donaldson GP, Shastri GG, Ann P, Ma L, Nagler CR, Ismagilov RF, Mazmanian SK, Hsiao EY. Indigenous bacteria from the gut microbiota regulate host serotonin biosynthesis. Cell. 
2015;161(2):264-76.

18. Koh A, De Vadder F, Kovatcheva-Datchary P, Bäckhed F. From dietary fiber to host physiology: shortchain fatty acids as key bacterial metabolites. Cell. 2016;165(6):1332-45.

19. Byndloss MX, Olsan EE, Rivera-Chávez F, Tiffany CR, Cevallos SA, Lokken KL, Torres TP, Byndloss AJ, Faber F, Gao Y, Litvak Y, Lopez CA, Xu G, Napoli E, Giulivi C, Tsolis RM, Revzin A, Lebrilla CB, Bäumler AJ. Microbiota-activated PPAR-y signaling inhibits dysbiotic Enterobacteriaceae expansion. Science. 2017;357(6351):570-5.

20. Anitha M, Vijay-Kumar M, Sitaraman SV, Gewirtz AT, Srinivasan S. Gut microbial products regulate murine gastrointestinal motility via Toll-like receptor 4 signaling. Gastroenterology. 2012;143(4):1006-16.e4.

21. Welihinda AA, Kaur M, Greene K, Zhai Y, Amento EP. The adenosine metabolite inosine is a functional agonist of the adenosine A2A receptor with a unique signaling bias. Cell Signal. 2016;28(6):552-60.

22. Mager LF, Burkhard R, Pett N, Cooke NCA, Brown K, Ramay H, Paik S, Stagg J, Groves RA, Gallo M, Lewis IA, Geuking MB, McCoy KD. Microbiome-derived inosine modulates response to checkpoint inhibitor immunotherapy. Science. 2020;369(6510):1481-9.

23. Ferreres F, Krsková Z, Gonçalves RF, Valentão P, Pereira JA, Dusek J, Martin J, Andrade PB. Free water-soluble phenolics profiling in barley (Hordeum vulgare L.). J Agric Food Chem. 2009;57(6):2405-9.

24. Yu YM, Chang WC, Chang CT, Hsieh CL, Tsai CE. Effects of young barley leaf extract and antioxidative vitamins on LDL oxidation and free radical scavenging activities in type 2 diabetes. Diabetes Metab. 2002;28(2):107-14.

25. Yamaura K, Tanaka R, Bi Y, Fukata H, Oishi N, Sato H, Mori C, Ueno K. Protective effect of young green barley leaf (Hordeum vulgare L.) on restraint stress-induced decrease in hippocampal brainderived neurotrophic factor in mice. Pharmacogn Mag. 2015;11(Suppl 1):S86-92.

26. Wirtz S, Popp V, Kindermann M, Gerlach K, Weigmann B, Fichtner-Feigl S, Neurath MF. Chemically induced mouse models of acute and chronic intestinal inflammation. Nat Protoc. 2017;12(7):1295309.

27. Bauer C, Duewell P, Mayer C, Lehr HA, Fitzgerald KA, Dauer M, Tschopp J, Endres S, Latz E, Schnurr M. Colitis induced in mice with dextran sulfate sodium (DSS) is mediated by the NLRP3 inflammasome. Gut. 2010;59(9):1192-9.

28. Shin NR, Whon TW, Bae JW. Proteobacteria: microbial signature of dysbiosis in gut microbiota. Trends Biotechnol 2015;33(9):496-503.

29. Sanders KM, Koh SD, Ro S, Ward SM. Regulation of gastrointestinal motility-insights from smooth muscle biology. Nat Rev Gastroenterol Hepatol. 2012;9(11):633-45.

30. Wei X, Yang Z, Rey FE, Ridaura VK, Davidson NO, Gordon JI, Semenkovich CF. Fatty acid synthase modulates intestinal barrier function through palmitoylation of mucin 2. Cell Host Microbe. 2012;11(2):140-52. 
31. Ledesma-Amaro R, Buey RM, Revuelta JL. Increased production of inosine and guanosine by means of metabolic engineering of the purine pathway in Ashbya gossypii. Microb Cell Fact. 2015;14:58.

32. He B, Hoang TK, Wang T, Ferris M, Taylor CM, Tian X, Luo M, Tran DQ, Zhou J, Tatevian N, Luo F, Molina JG, Blackburn MR, Gomez TH, Roos S, Rhoads JM, Liu Y. Resetting microbiota by Lactobacillus reuteri inhibits $T$ reg deficiency-induced autoimmunity via adenosine A2A receptors. $J$ Exp Med. 2017, 214(1):107-123.

33. Kappelman MD, Rifas-Shiman SL, Porter CQ, Ollendorf DA, Sandler RS, Galanko JA, Finkelstein JA. Direct health care costs of Crohn's disease and ulcerative colitis in US children and adults. Gastroenterology. 2008;135(6):1907-13.

34. Hooper LV, Macpherson AJ. Immune adaptations that maintain homeostasis with the intestinal microbiota. Nat Rev Immunol. 2010;10(3):159-69.

35. Hooper LV, Littman DR, Macpherson AJ. Interactions between the microbiota and the immune system. Science. 2012;336(6086):1268-73.

36. Honda K, Littman DR. The microbiota in adaptive immune homeostasis and disease. Nature. 2016;535(7610):75-84.

37. Ni J, Wu GD, Albenberg L, Tomov VT. Gut microbiota and IBD: causation or correlation? Nat Rev Gastroenterol Hepatol. 2017;14(10):573-84.

38. Ott SJ, Musfeldt M, Wenderoth DF, Hampe J, Brant O, Fölsch UR, Timmis KN, Schreiber S. Reduction in diversity of the colonic mucosa associated bacterial microflora in patients with active inflammatory bowel disease. Gut. 2004;53(5):685-93.

39. Manichanh C, Rigottier-Gois L, Bonnaud E, Gloux K, Pelletier E, Frangeul L, Nalin R, Jarrin C, Chardon P, Marteau P, Roca J, Dore J. Reduced diversity of faecal microbiota in Crohn's disease revealed by a metagenomic approach. Gut. 2006;55(2):205-11.

40. Lupp C, Robertson ML, Wickham ME, Sekirov I, Champion OL, Gaynor EC, Finlay BB. Host-mediated inflammation disrupts the intestinal microbiota and promotes the overgrowth of Enterobacteriaceae. Cell Host Microbe. 2007;2(3):204.

41. Johansson ME, Gustafsson JK, Holmén-Larsson J, Jabbar KS, Xia L, Xu H, Ghishan FK, Carvalho FA, Gewirtz AT, Sjövall H, Hansson GC. Bacteria penetrate the normally impenetrable inner colon mucus layer in both murine colitis models and patients with ulcerative colitis. Version 2. Gut. 2014;63(2):281-91.

42. Barros LL, Farias AQ, Rezaie A. Gastrointestinal motility and absorptive disorders in patients with inflammatory bowel diseases: Prevalence, diagnosis and treatment. World J Gastroenterol. 2019;25(31):4414-26.

43. Dubuquoy L, Jansson EA, Deeb S, Rakotobe S, Karoui M, Colombel JF, Auwerx J, Pettersson S, Desreumaux P. Impaired expression of peroxisome proliferator-activated receptor gamma in ulcerative colitis. Gastroenterology. 2003;124(5):1265-76.

44. Lefebvre M, Paulweber B, Fajas L, Woods J, McCrary C, Colombel JF, Najib J, Fruchart JC, Datz C, Vidal H, Desreumaux P, Auwerx J. Peroxisome proliferator-activated receptor gamma is induced 
during differentiation of colon epithelium cells. J Endocrinol. 1999;162(3):331-40.

45. Hontecillas R, Bassaganya-Riera J. Peroxisome proliferator-activated receptor gamma is required for regulatory CD4+ T cell-mediated protection against colitis. J Immunol. 2007;178(5):2940-9.

46. Peyrin-Biroulet L, Beisner J, Wang G, Nuding S, Oommen ST, Kelly D, Parmentier-Decrucq E, Dessein R, Merour E, Chavatte P, Grandjean T, Bressenot A, Desreumaux P, Colombel JF, Desvergne B, Stange EF, Wehkamp J, Chamaillard M. Peroxisome proliferator-activated receptor gamma activation is required for maintenance of innate antimicrobial immunity in the colon. Proc Natl Acad Sci U S A. 2010;107(19):8772-7.

47. Tomas J, Mulet C, Saffarian A, Cavin JB, Ducroc R, Regnault B, Kun Tan C, Duszka K, Burcelin R, Wahli W, Sansonetti PJ, Pédron T. High-fat diet modifies the PPAR-ץ pathway leading to disruption of microbial and physiological ecosystem in murine small intestine. Proc Natl Acad Sci U S A. 2016;113(40):E5934-43.

48. Su CG, Wen X, Bailey ST, Jiang W, Rangwala SM, Keilbaugh SA, Flanigan A, Murthy S, Lazar MA, Wu GD. A novel therapy for colitis utilizing PPAR-gamma ligands to inhibit the epithelial inflammatory response. J Clin Invest. 1999;104(4):383-9.

49. Desreumaux P, Dubuquoy L, Nutten S, Peuchmaur M, Englaro W, Schoonjans K, Derijard B, Desvergne B, Wahli W, Chambon P, Leibowitz MD, Colombel JF, Auwerx J. Attenuation of colon inflammation through activators of the retinoid $X$ receptor $(\mathrm{RXR}) /$ peroxisome proliferator-activated receptor gamma (PPARgamma) heterodimer. A basis for new therapeutic strategies. J Exp Med. 2001;193(7):827-38.

50. Lewis JD, Lichtenstein GR, Stein RB, Deren JJ, Judge TA, Fogt F, Furth EE, Demissie EJ, Hurd LB, Su CG, Keilbaugh SA, Lazar MA, Wu GD. An open-label trial of the PPAR-gamma ligand rosiglitazone for active ulcerative colitis. Am J Gastroenterol. 2001;96(12):3323-8.

51. Rousseaux C, Lefebvre B, Dubuquoy L, Lefebvre P, Romano O, Auwerx J, Metzger D, Wahli W, Desvergne B, Naccari GC, Chavatte P, Farce A, Bulois P, Cortot A, Colombel JF, Desreumaux P. Intestinal antiinflammatory effect of 5-aminosalicylic acid is dependent on peroxisome proliferatoractivated receptor-gamma. J Exp Med. 2005;201(8):1205-15.

52. Bassaganya-Riera J, Reynolds K, Martino-Catt S, Cui Y, Hennighausen L, Gonzalez F, Rohrer J, Benninghoff AU, Hontecillas R. Activation of PPAR gamma and delta by conjugated linoleic acid mediates protection from experimental inflammatory bowel disease. Gastroenterology. 2004;127(3):777-91.

53. Alonso L, Cuesta EP, Gilliland SE. Production of free conjugated linoleic acid by Lactobacillus acidophilus and Lactobacillus casei of human intestinal origin. J Dairy Sci. 2003;86(6):1941-6.

54. Alex S, Lange K, Amolo T, Grinstead JS, Haakonsson AK, Szalowska E, Koppen A, Mudde K, Haenen D, Al-Lahham S, Roelofsen H, Houtman R, van der Burg B, Mandrup S, Bonvin AM, Kalkhoven E, Müller M, Hooiveld GJ, Kersten S. Short-chain fatty acids stimulate angiopoietin-like 4 synthesis in human colon adenocarcinoma cells by activating peroxisome proliferator-activated receptor $\mathrm{Y}$. Mol Cell Biol. 2013;33(7):1303-16. 
55. Asahara T, Mori Y, Zakataeva NP, Livshits VA, Yoshida K, Matsuno K. Accumulation of gene-targeted Bacillus subtilis mutations that enhance fermentative inosine production. Appl Microbiol Biotechnol. 2010;87(6):2195-207.

56. Sheremet AS, Gronskiy SV, Akhmadyshin RA, Novikova AE, Livshits VA, Shakulov RS, Zakataeva NP. Enhancement of extracellular purine nucleoside accumulation by Bacillus strains through genetic modifications of genes involved in nucleoside export. J Ind Microbiol Biotechnol. 2011;38(1):65-70.

57. Peifer S, Barduhn T, Zimmet S, Volmer DA, Heinzle E, Schneider K. Metabolic engineering of the purine biosynthetic pathway in Corynebacterium glutamicum results in increased intracellular pool sizes of IMP and hypoxanthine. Microb Cell Fact. 2012;11:138.

58. Shimaoka M, Takenaka Y, Kurahashi O, Kawasaki H, Matsui H. Effect of amplification of desensitized purF and prs on inosine accumulation in Escherichia coli. J Biosci Bioeng. 2007;103(3):255-61.

59. Haskó G, Sitkovsky MV, Szabó C. Immunomodulatory and neuroprotective effects of inosine. Trends Pharmacol Sci. 2004;25(3):152-7.

60. Murthy SN, Cooper HS, Shim H, Shah RS, Ibrahim SA, Sedergran DJ. Treatment of dextran sulfate sodium-induced murine colitis by intracolonic cyclosporin. Dig Dis Sci. 1993 Sep;38(9):1722-34.

61. Rachmilewitz D, Karmeli F, Takabayashi K, Hayashi T, Leider-Trejo L, Lee J, Leoni LM, Raz E. Immunostimulatory DNA ameliorates experimental and spontaneous murine colitis. Gastroenterology. 2002, 122(5):1428-41.

62. Love MI, Huber W, Anders S. Moderated estimation of fold change and dispersion for RNA-seq data with DESeq2. Genome Biol. 2014;15(12):550.

\section{Figures}


a

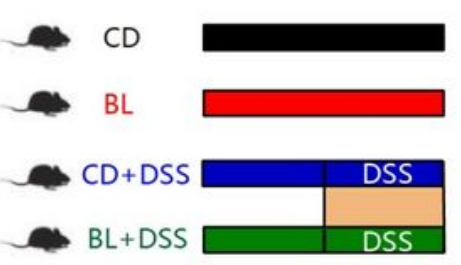

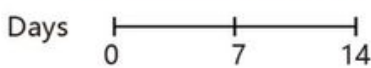

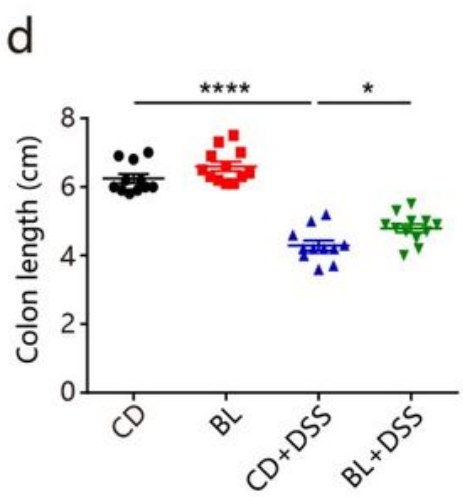

b

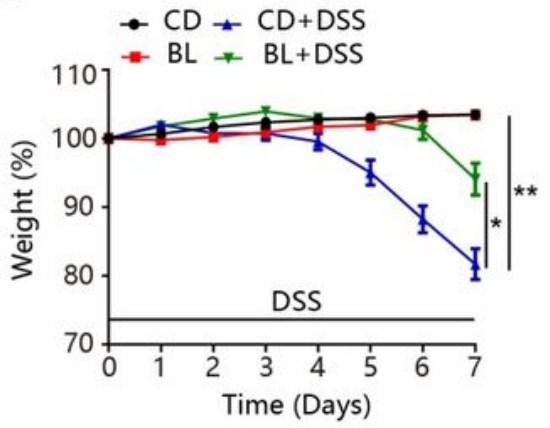

e

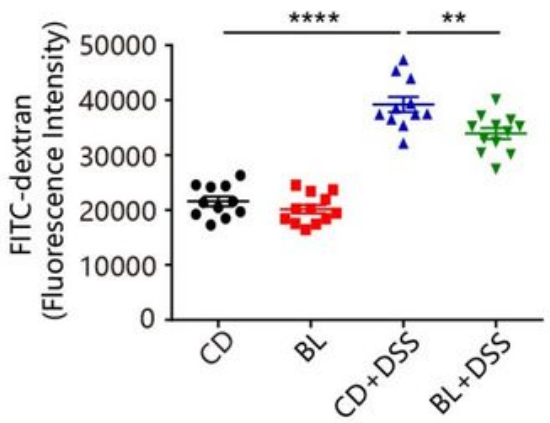

C

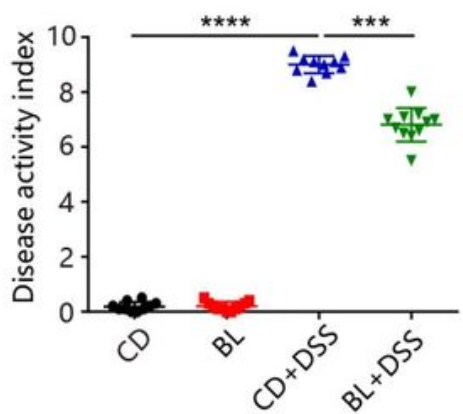

f

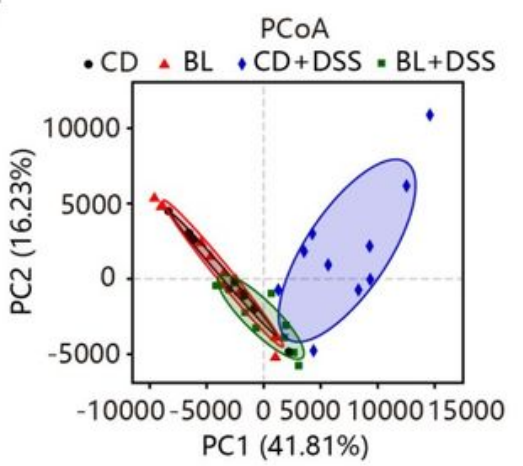

g
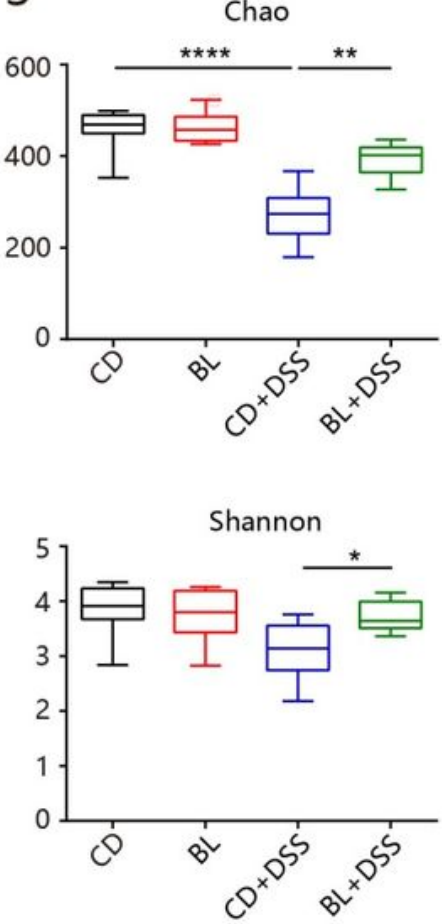

$\mathrm{h}$

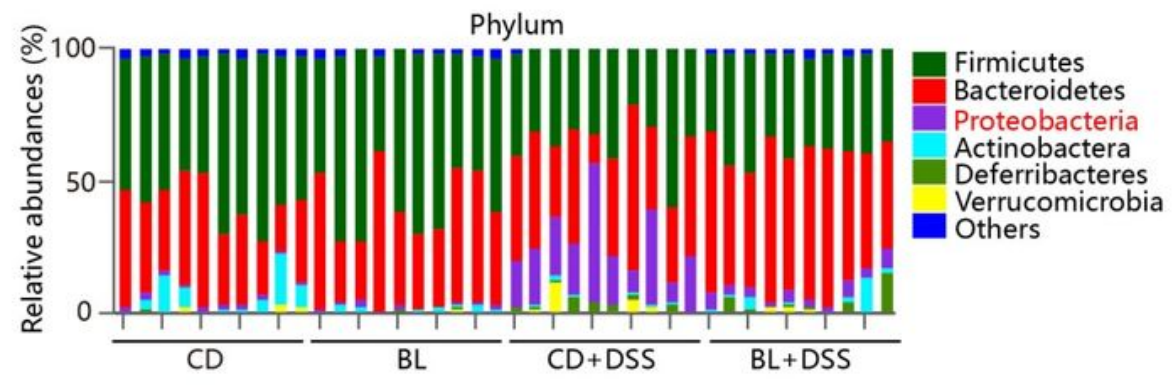

i

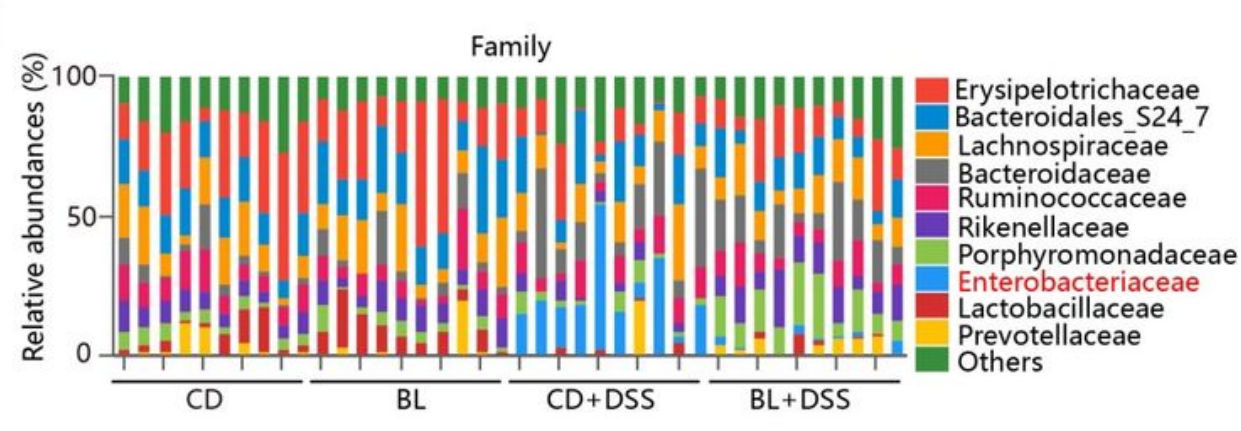

Figure 1

Barley leaf (BL) attenuates dextran sulphate sodium (DSS)-induced colitis and gut microbiota dysbiosis. (a-i) Mice were fed with a standard chow diet (CD) or an isocaloric BL-supplemented diet for two weeks. Colitis was induced by administering 2.5\% DSS dissolved in drinking water for 7 days. (a) Study design of in vivo mouse experiment. (b) Percentage body weight change, (c) diseases activity scores, (d) colon lengths and (e) intestinal permeability of the CD- and BL-fed mice with or without DSS treatment $(n=10$ - 
12). (f) Weighted UniFrac Principal coordinate analysis (PCoA) plot of the gut microbiota composition at the operational taxonomic unit (OTU) level from different mouse groups $(\mathrm{n}=10)$. ( $\mathrm{g}$ ) Alpha diversity analysis of gut bacterial richness (Chao1 index) and diversity (Shannon index) from different mouse groups $(n=10)$. (h) Taxonomic distributions of gut bacterial composition at phylum level $(n=10)$. (i) Taxonomic distributions of gut bacterial composition at family level $(n=10)$. Data are pooled from three independent experiments (a-e). Data are mean \pm SEM. ${ }^{*} \mathrm{P}<0.05$, ${ }^{\star *} \mathrm{P}<0.01,{ }^{\star \star *} \mathrm{P}<0.001$ and ${ }^{\star \star \star *} \mathrm{P}<$ 0.0001 . For body weight change, a repeated measure two-way analysis of variance (ANOVA) was performed and the rest of the statistics was performed with one-way ANOVA followed by Tukey's multiple comparison's test.

a

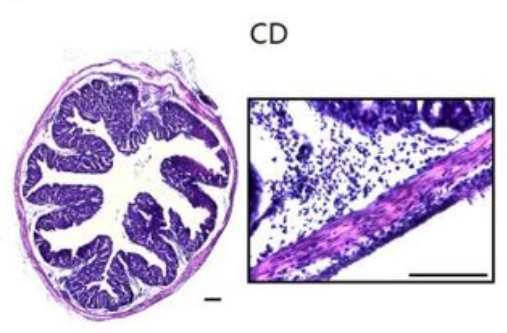

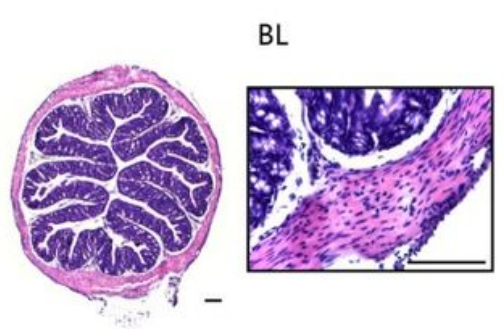

g
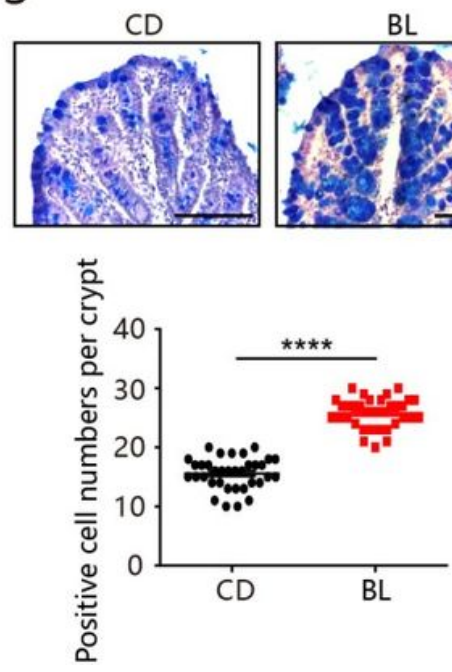
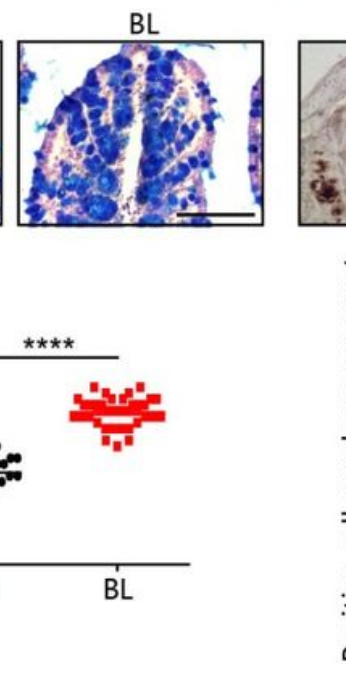

b

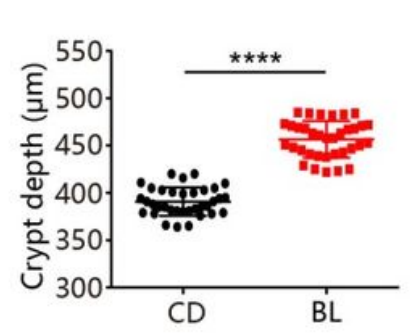

C

d

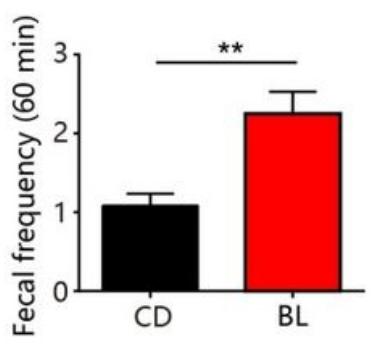

e

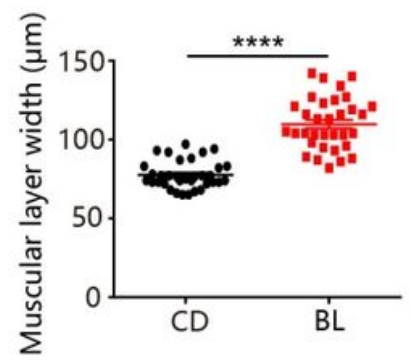

$\mathrm{h}$
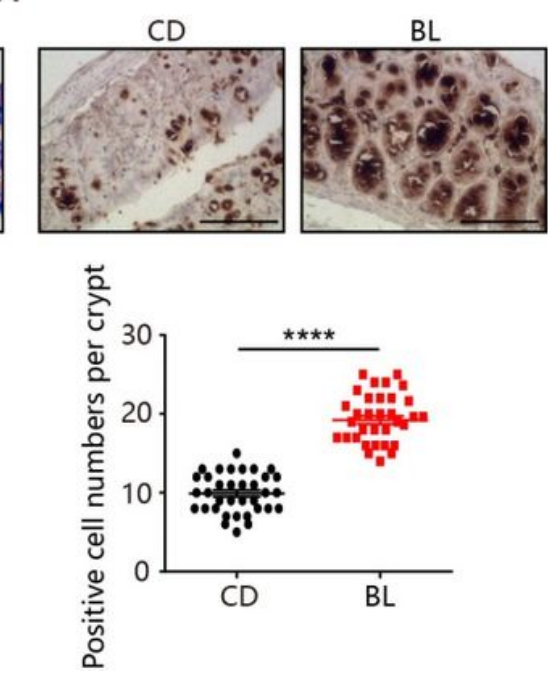

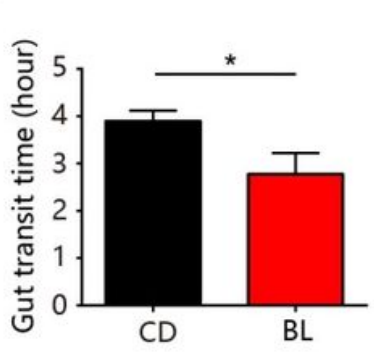

i f
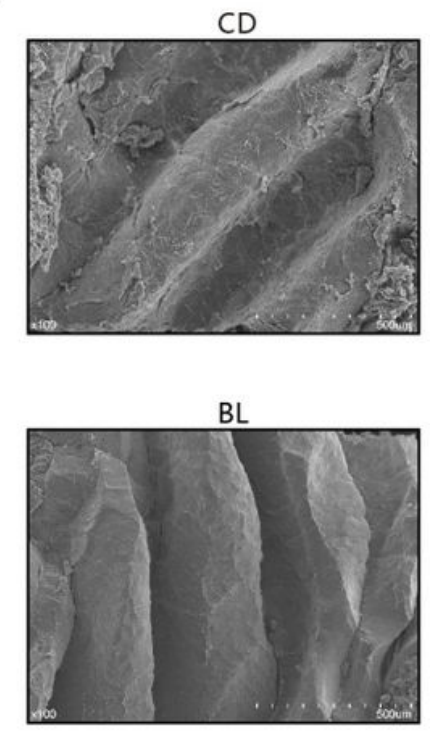
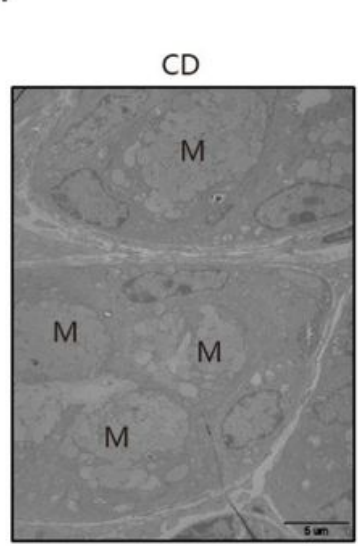

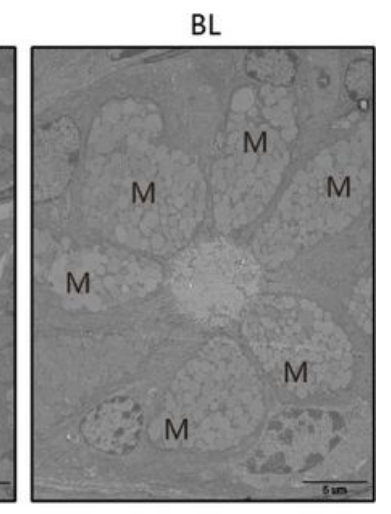

\section{Figure 2}

Barley leaf $(B L)$ enhances colonic motility and improves mucosal barrier function. (a-i) Mice were fed with a standard chow diet (CD) or an isocaloric BL-supplemented diet for two weeks. (a) Representative images of hematoxylin and eosin-stained colonic sections. The right panels were enlarged from the left 
panels. Scale bar $=200 \mu \mathrm{m}$ (left) and $100 \mu \mathrm{m}$ (right). (b) Crypt height and (c) muscular layer width in the colon of CD- and BL-fed mice were quantified $(n=12)$. (d) Fecal pellet output and (e) gut transit time of $C D$ - and BL-fed mice were measured $(n=8)$. ( $f$ ) Representative images of scanning electron microscopy of colonic mucosal surface. Scale bar $=500 \mu \mathrm{m}$. (g) Representative images of alcian blue-stained colonic sections and the number of mucusproducing goblet cells was quantified $(n=12)$. Scale bar $=100 \mu \mathrm{m}$. $(\mathrm{h})$ Representative images of Muc2-stained colon sections and the number of Muc-2 positive goblet cells was quantified $(n=12)$. Scale bar $=100 \mu$ m. (i) Representative images of transmission electron microscopy of the mucin granules $(M)$ in colonic goblet cells. Scale bar $=5 \mu \mathrm{m}$. Data are pooled from three independent experiments. Data are mean \pm SEM. ${ }^{*} \mathrm{P}<0.05$, ${ }^{\star *} \mathrm{P}<0.01$ and ${ }^{\star *} * \star \mathrm{P}<0.0001$. Statistical analysis was performed using Student's $t$ test. 
a

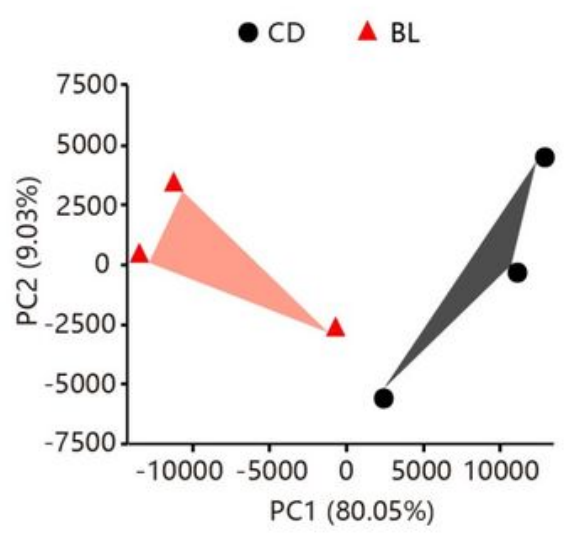

C

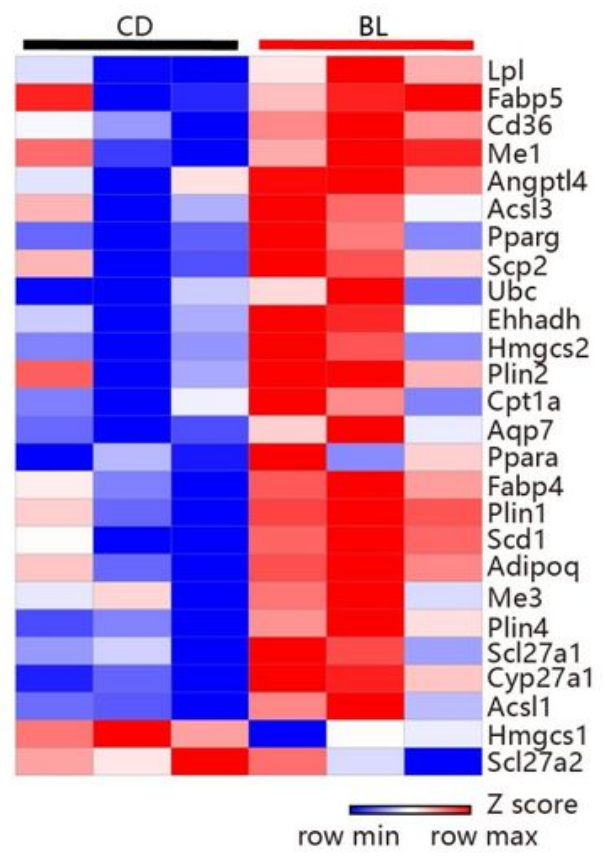

e

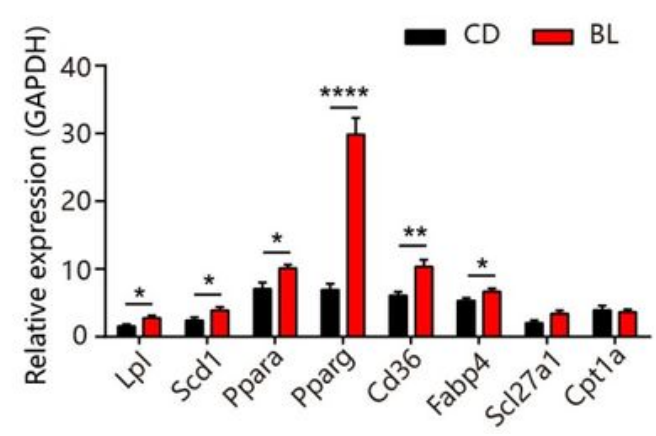

b

KEGG enrichement analysis

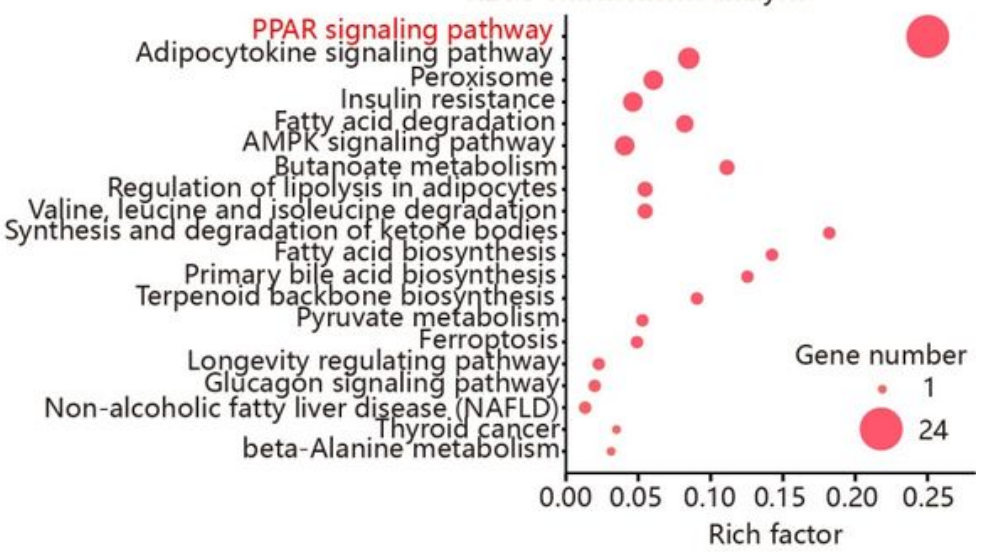

d

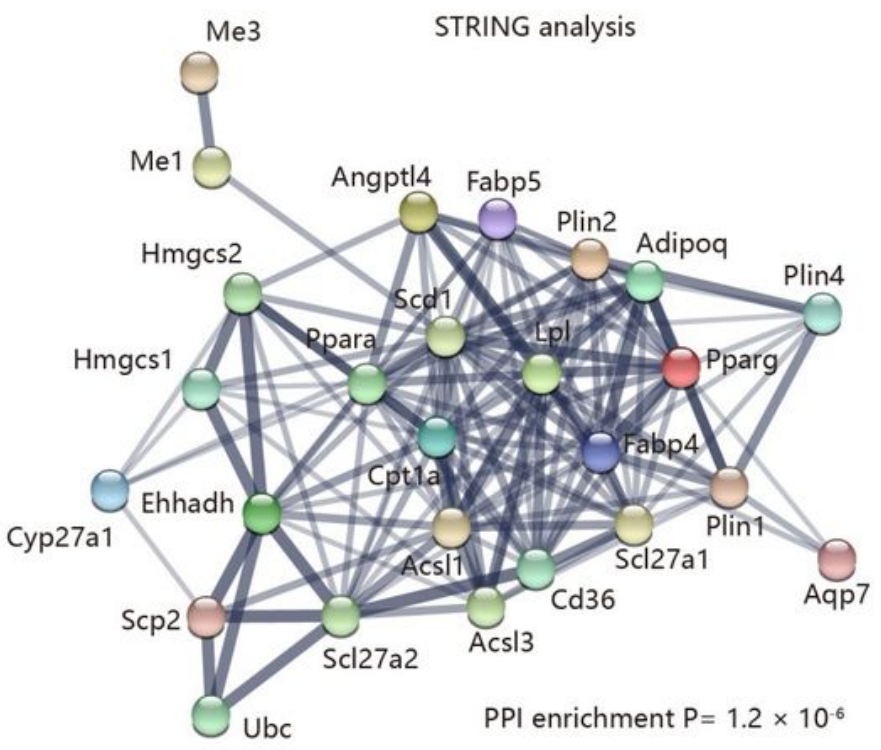

f
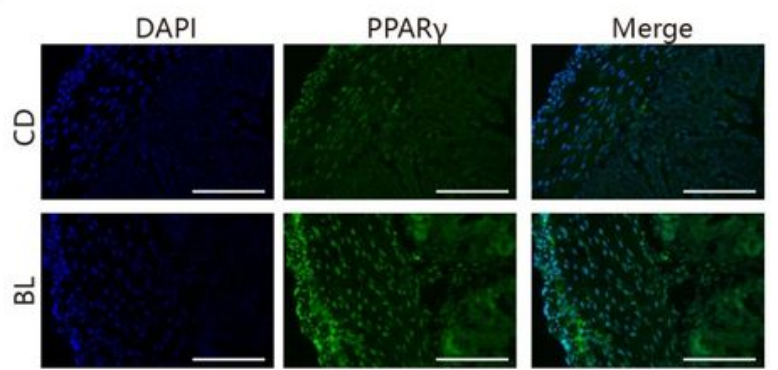

\section{Figure 3}

Barley leaf (BL) alters gene expression in mouse colon. (a-f) Mice were fed a standard chow diet (CD) or an isocaloric BL-supplemented diet for two weeks $(n=3)$. (a) Principle component analysis (PCA) of transcriptional profiling of the mouse colonic tissues. Permutational multivariate analysis of variance (PERMANOVA): $F=6.00, \mathrm{Df}=2, \mathrm{P}=0.017$. (b) Kyoto Encyclopedia of Genes and Genomes (KEGG) pathway enrichment analysis of the most significantly changed pathways. (c) Heat map of differentially 
expressed genes in peroxisome proliferators-activated receptor (PPAR) signaling pathway. Genes with fold changes of $>1$ and false discovery rates of $<0.05$ were considered to be differentially expressed. (d) Search tool for recurring instances of neighbouring genes (STRING) network visualization of the genes in differentially expressed genes in PPAR signaling pathway. Edges represent protein-protein associations. (e) Real-time PCR assay for the differentially expressed genes in PPAR signaling pathway $(n=10)$. (f) Immunofluorescent analysis of PPARY (green) in mouse colonic sections. Nuclei were stained with DAPI (blue). Scale bar $=100 \mu \mathrm{m}$. Data are pooled from three independent experiments (e). Data are representative of two independent experiments (f). Data are mean $\pm S E M$. ${ }^{\star} P<0.05, * \star P<0.01$ and $* \star \star \star P$ $<0.0001$. Statistical analysis was performed using Student's $t$ test. 
a

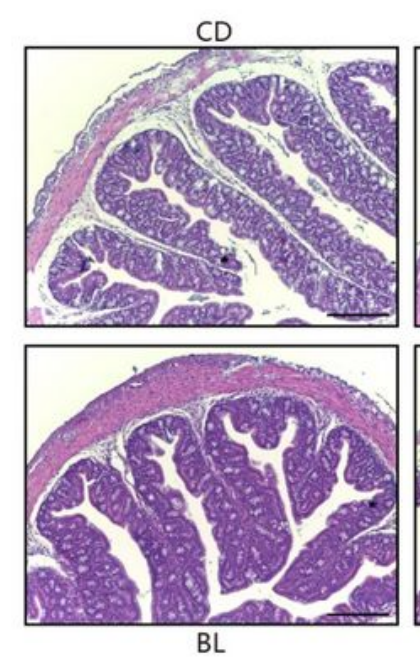

f
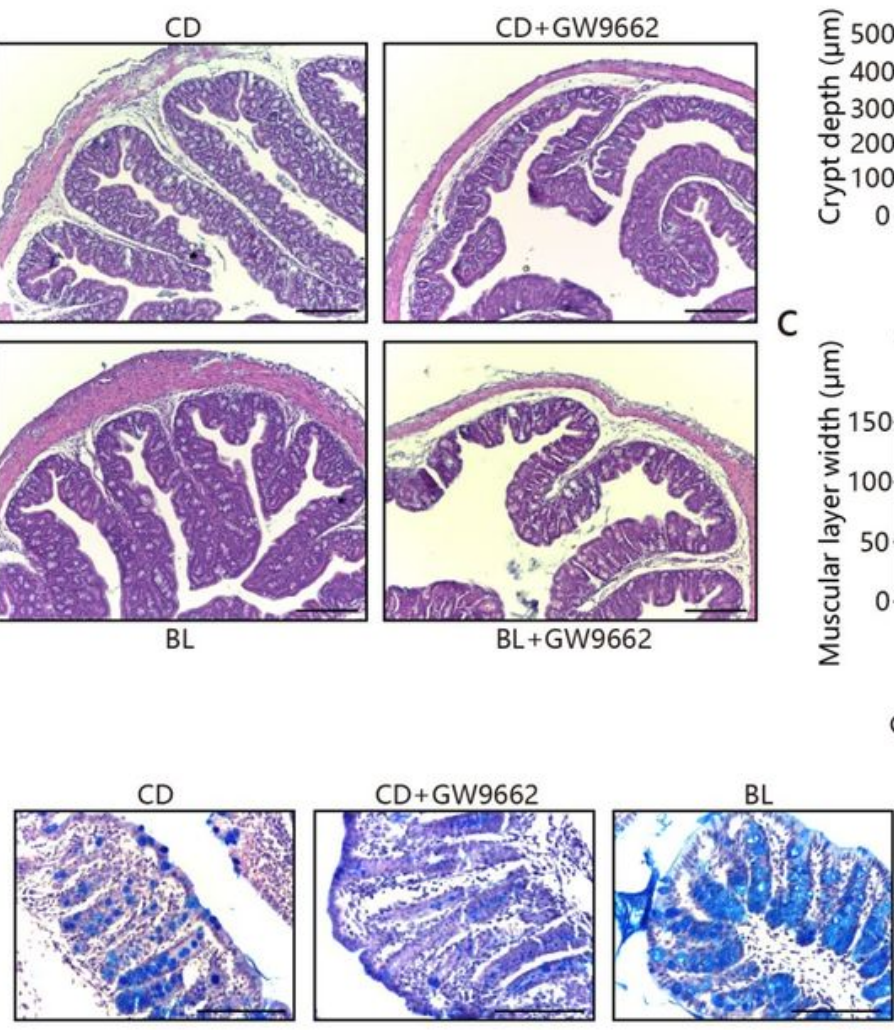

\section{$c$}
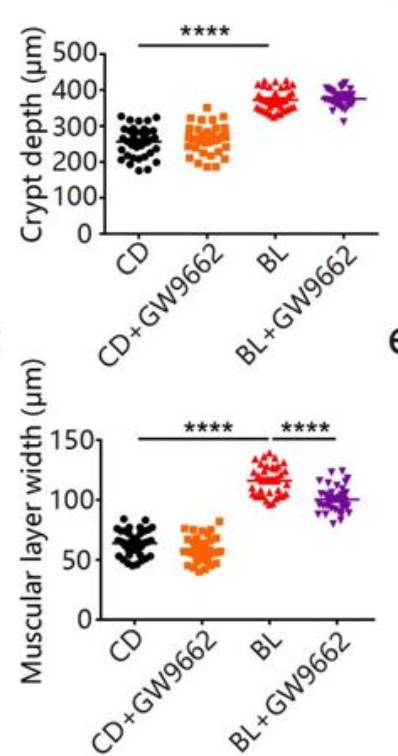

d
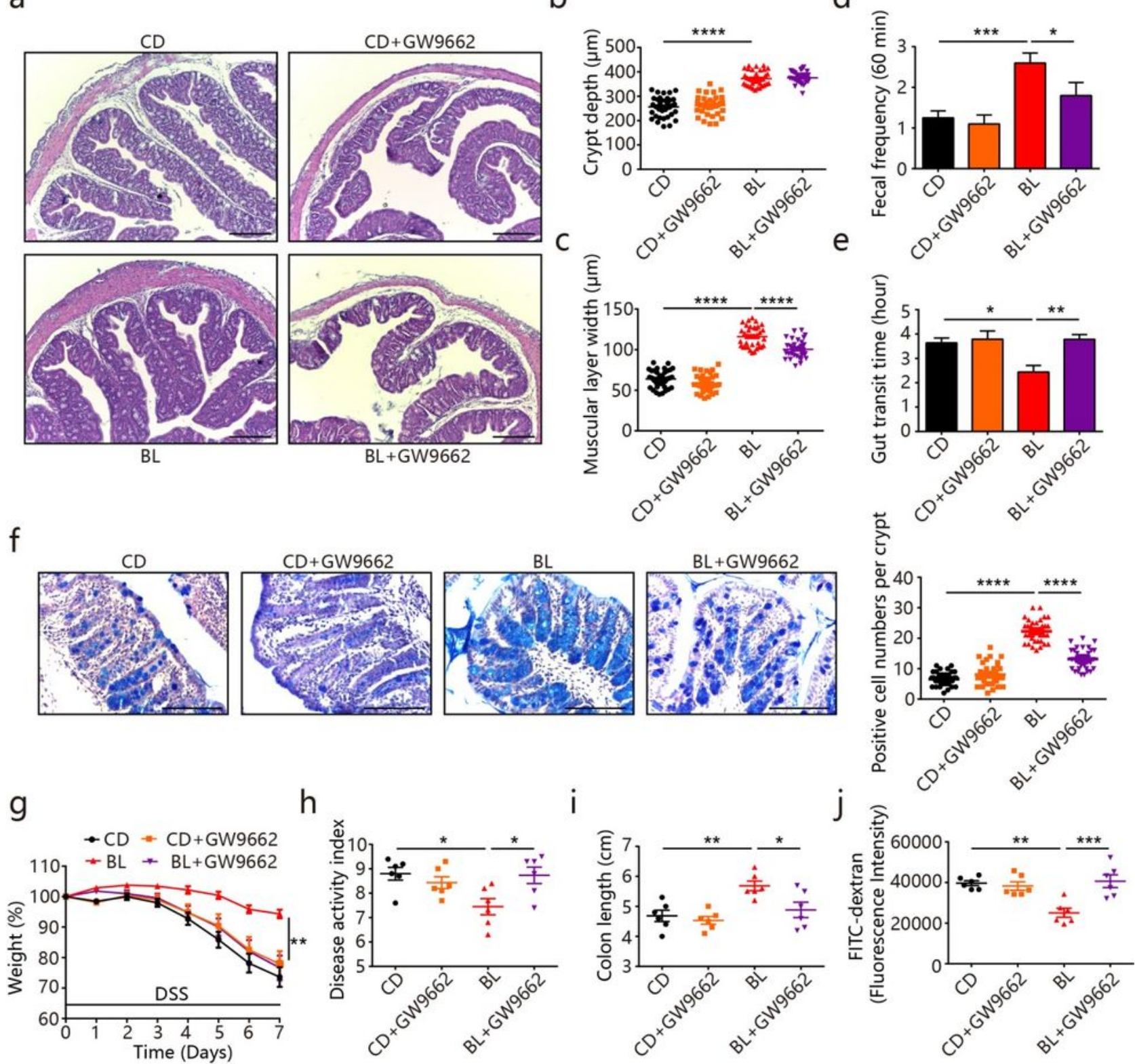

$\mathrm{k}$
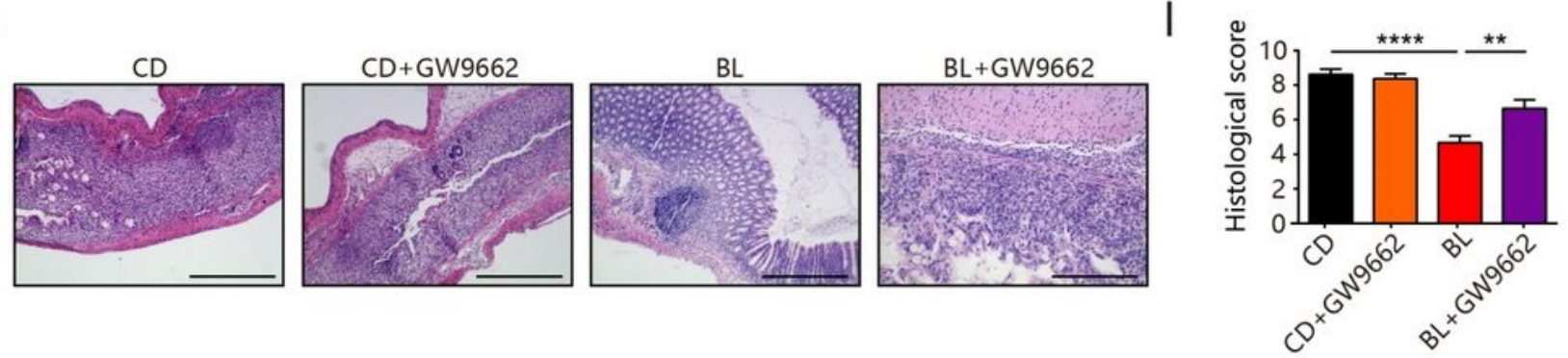

Figure 4

Barley leaf (BL) improves intestinal functions though PPARy signaling activation. (a-l) Mice were fed a chow diet (CD) or an isocaloric BL-supplemented diet for two weeks. PPARy antagonist GW9662 was administered with $3 \mathrm{mg} / \mathrm{kg} /$ day intragastrically. Colitis was induced by administering 2.5\% DSS dissolved in drinking water for 7 days. (a) Representative images of hematoxylin and eosin-stained colonic sections. Scale bar $=100 \mu \mathrm{m}$. (b) Crypt height and (c) muscular layer width in the colon of mice from 
different mouse groups were quantified $(n=8)$. (d) Fecal pellet output and (e) gut transit time from different mouse groups were measured $(n=8)$. (f) Representative images of alcian blue-stained colonic sections and the number of mucusproducing goblet cells was quantified $(\mathrm{n}=8)$. Scale bar $=200 \mu \mathrm{m}$. $(\mathrm{g})$ Percentage body weight change, (h) diseases activity scores (i) colon lengths and (j) intestinal permeability from different mouse groups were measured $(n=6)$. (k) Representative images of hematoxylin and eosin-stained colonic sections and (I) histological scores. Scale bar $=200 \mu \mathrm{m}$. Data are pooled from three independent experiments. Data are mean $\pm S E M$. ${ }^{*} P<0.05, * * P<0.01, * \star \star P<0.001$ and ${ }^{* \star * *} P<0.0001$. For body weight change, a repeated measure two-way analysis of variance (ANOVA) was performed and the rest of the statistics was performed with one-way ANOVA followed by Tukey's multiple comparison's test. 
a

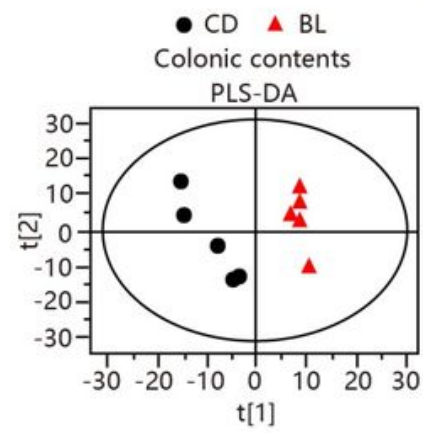

b

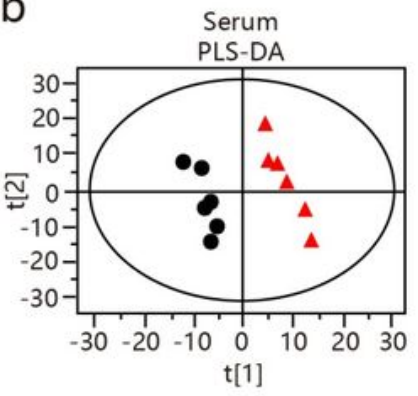

e

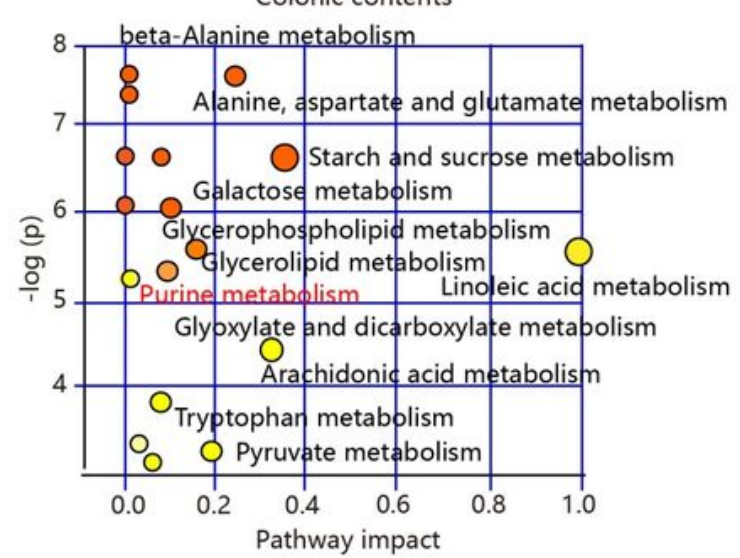

Colonic contents

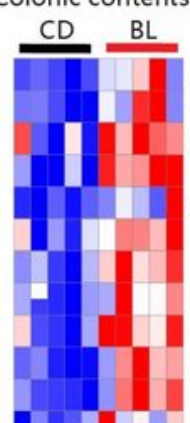

2-Deoxyguanosine

2'-Deoxyinosine

Glutaric acid

alpha-Tocopherol

Arachidonic acid

beta-Sitosterol

Campesterol

Linoleic acid

5-Hydroxyindoleacetic acid

Lyxose

4-Hydroxyphenylpropionic acid

Sucrose

Glucose

Glycerol 3-phosphate

Guanosine

5-Hydroxytryptamine

Aspartic acid

Glyceric acid

Phosphate

Pyruvic acid

Adenine

Spermidine

d

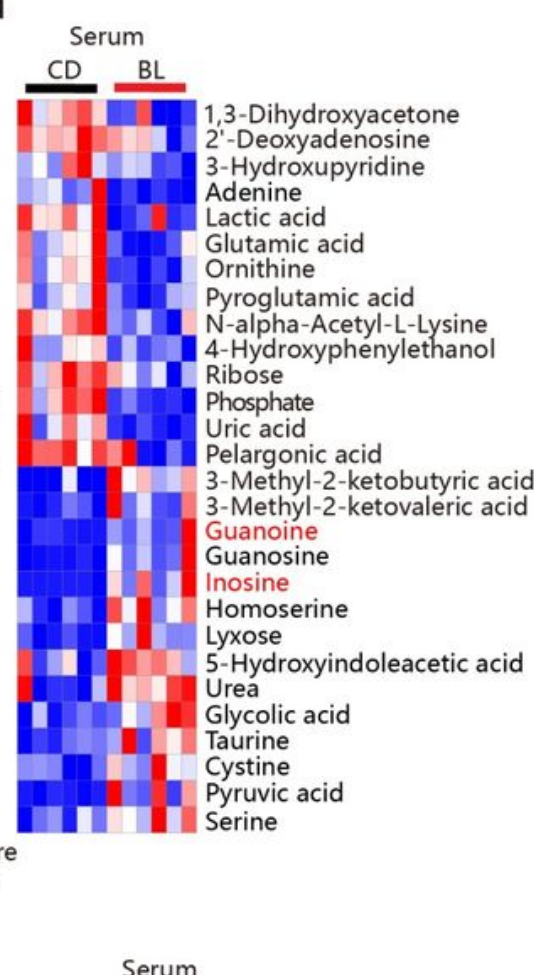

f

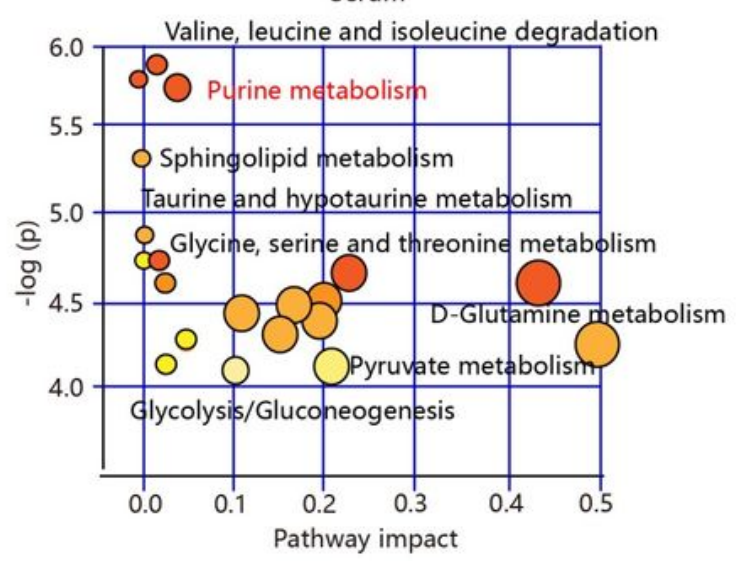

h

g

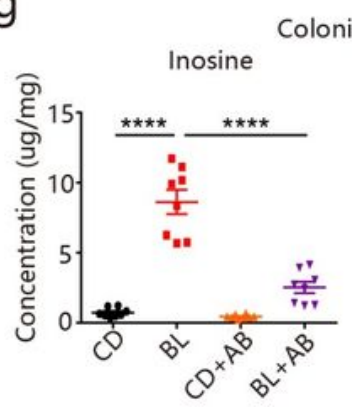

Colonic contents

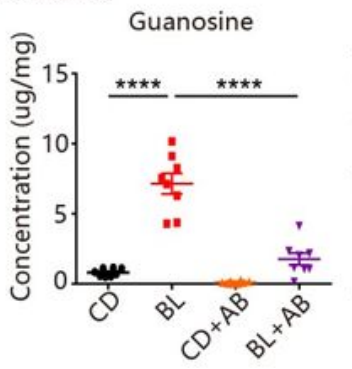

Serum
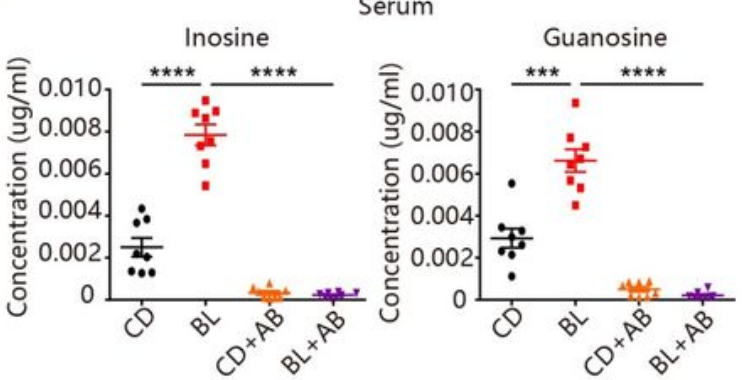

\section{Figure 5}

Barley leaf (BL) alters metabolic profiling and enriched microbiota-derived purine metabolites. (a-h) Mice were fed with a standard chow diet (CD) or an isocaloric BL-supplemented diet for two weeks. (a and b) Partial least-squares discriminant analysis (PLS-DA) of metabolomic profiles in colonic contents $(n=5)$ and serum ( $n=6)$ of CD- and BL-fed mice. ( $c$ and d) Heat map of significantly altered metabolites in colonic contents $(n=5)$ and serum $(n=6)$ of CD- and BL-fed mice. Metabolites with the Variable 
importance in the projection (VIP) value of PLS-DA model $>1$ and the $\mathrm{P}$ values of Student's $\mathrm{t}$ test $<0.05$ were considered to be significantly different. (e and f) Pathway enrichment analysis of the significantly altered metabolites in colonic contents and serum of CD- and BL-fed mice. $x$ Axis represents the pathway impact, and y axis represents the pathway enrichment. ( $g$ and $h$ ) Concentrations of inosine and guanosine in colonic contents and serum of CD- and BL-fed mice $(n=8) . A B$, antibiotics. Data are pooled from three independent experiments ( $g$ and $h$ ). Data are mean \pm SEM. ${ }^{\star \star *} P<0.001$ and ${ }^{\star \star \star *} P(0.0001$. Statistical analysis was performed by one-way ANOVA followed by Tukey's multiple comparison's test.

a

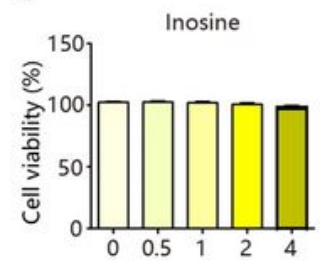

b
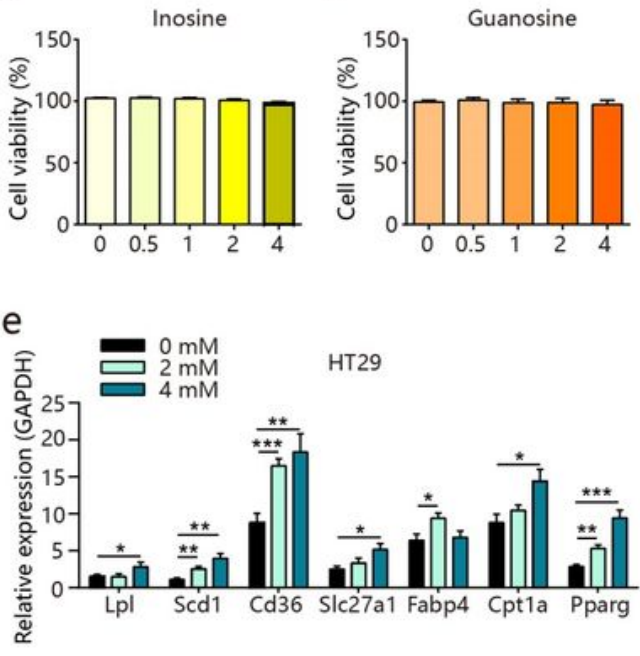

c
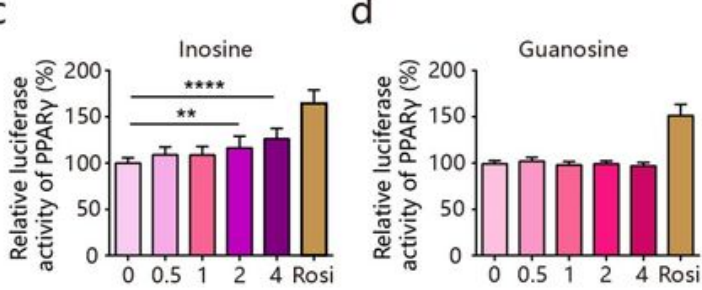

f

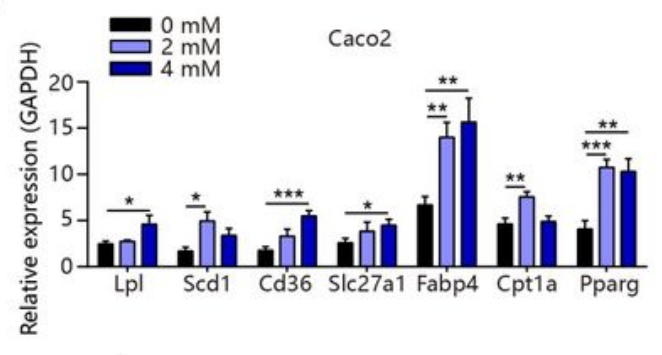

g

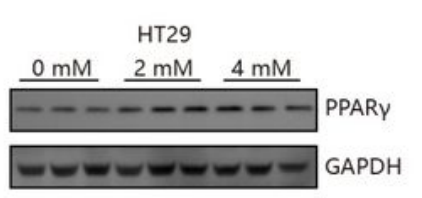

h
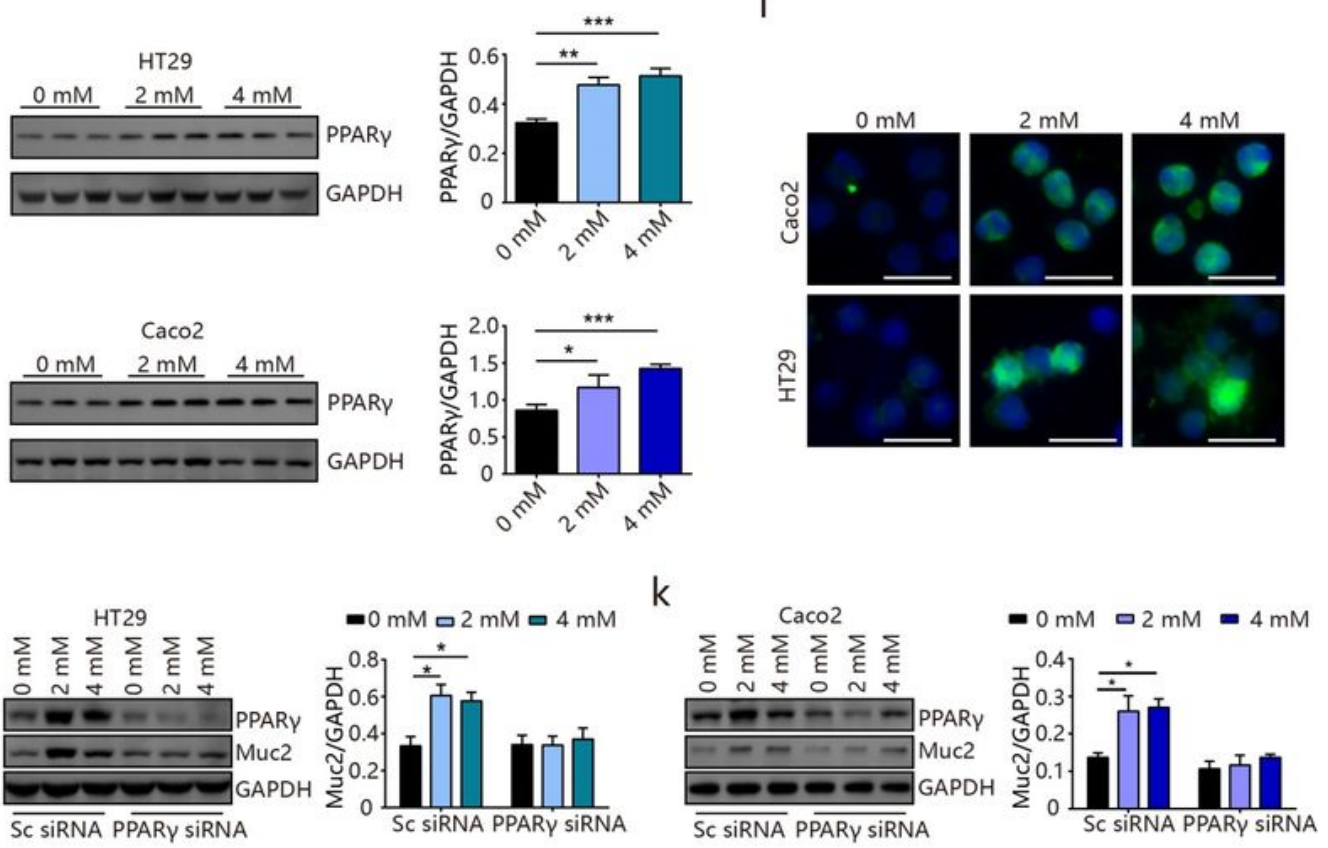

$\overline{\text { SC SIRNA }}$ PPARY SIRNA

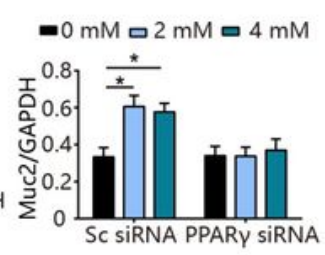

I
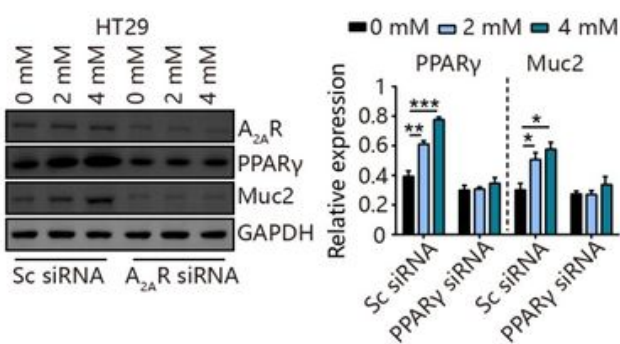

m
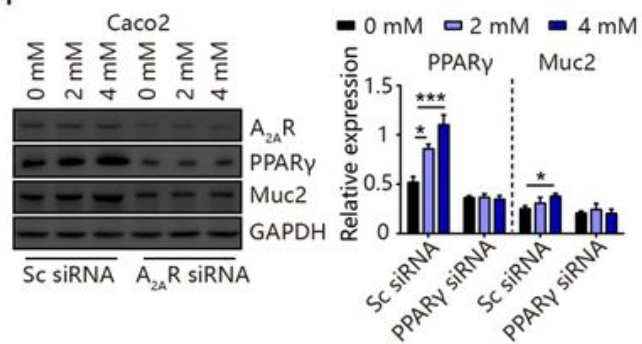


\section{Figure 6}

Inosine, but not guanosine, activates PPARy signaling pathway in human colon epithelial cells. ( $a$ and $b$ ) HT29 cells were treated with inosine $(0.5,1,2$ and $4 \mathrm{mM})$ or guanosine $(0.5,1,2$ and $4 \mathrm{mM})$ for $24 \mathrm{~h}$ and cell viability analysis was measured by CCK-8 assay. (c and d) HT29 cells were treated with inosine (0.5, 1,2 and $4 \mathrm{mM}$ ) or guanosine $(0.5,1,2$ and $4 \mathrm{mM}$ ) or $10 \mu \mathrm{M}$ rosiglitazone (Rosi) for $24 \mathrm{~h}$ and PPARy activity was measured by luciferase reporter gene assay. (e and f) HT29 and Caco2 cells were treated with inosine ( 2 and $4 \mathrm{mM}$ ) for $24 \mathrm{~h}$ and the expression of genes in PPARy signaling pathway was examined by real-time PCR assay. ( $\mathrm{g}$ and $\mathrm{h}$ ) HT29 and Caco2 cells were treated with inosine ( 2 and 4 $\mathrm{mM}$ ) for $24 \mathrm{~h}$ and the protein level of PPARy was examined by western blot. (i) HT29 and Caco2 cells were treated with inosine ( 2 and $4 \mathrm{mM}$ ) for $24 \mathrm{~h}$ and the protein level of PPARY (green) was examined by immunofluorescence analysis. Nuclei were stained with DAPI (blue). Scale bar $=20 \mu \mathrm{m}$. (j and k) HT29 and Caco2 cells were treated with inosine ( 2 and $4 \mathrm{mM}$ ) for $24 \mathrm{~h}$ and PPARy was knocked down using siRNA. The protein levels of PPARy and Muc2 were examined by western blot. (I and m) HT29 and Caco2 cells were treated with inosine ( 2 and $4 \mathrm{mM}$ ) for $24 \mathrm{~h}$ and A2AR was knocked down using siRNA. The protein levels of A2AR, PPARy and Muc2 were examined by western blot. Scrambled (Sc) siRNA transfections were used as controls. Immunoblots were quantified using Image $\mathrm{J}$ software. Data are pooled from three independent experiments (a-f). Data are representative of two independent experiments $(\mathrm{g}-\mathrm{m})$. Data are mean \pm SEM. ${ }^{*} \mathrm{P}<0.05,{ }^{*} \mathrm{P}<0.01,{ }^{*} * \mathrm{P}<0.001$ and ${ }^{* \star * *} \mathrm{P}<0.0001$. Statistical analysis was performed using Student's t test. 
a

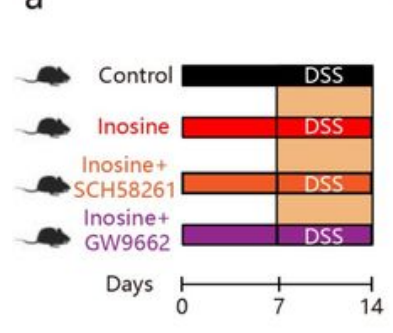

b

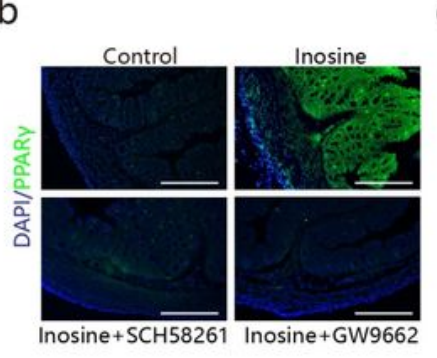

C

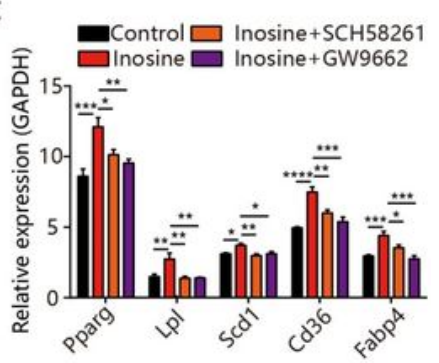

d
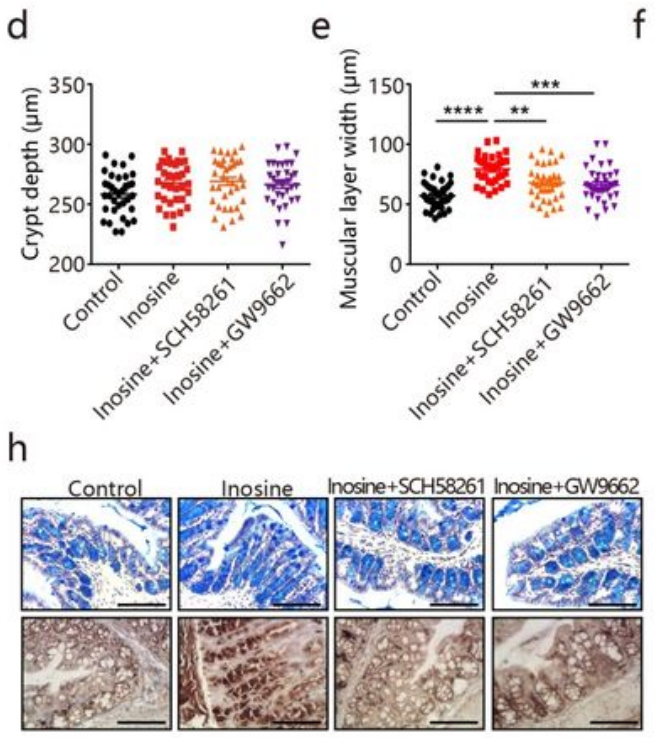

$f$

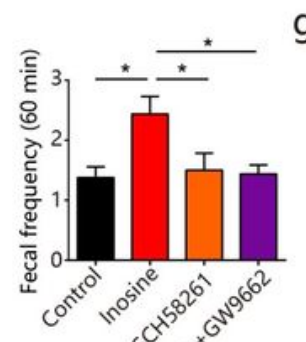

$\mathrm{g}$

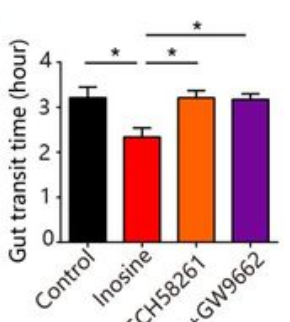

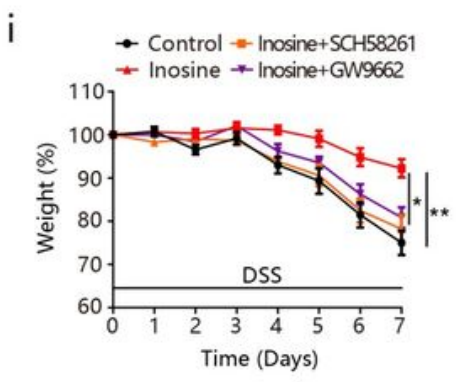

|

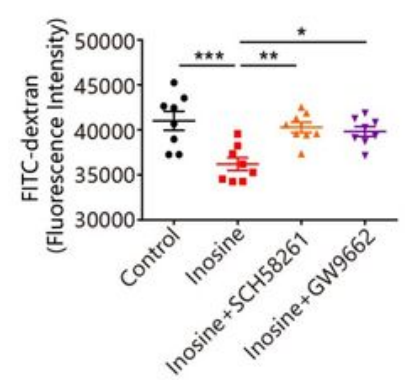

j

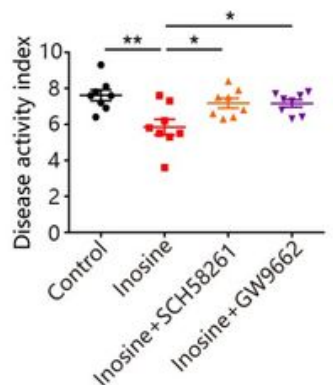

m

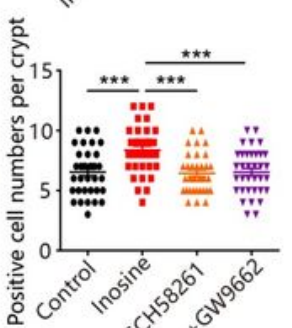

k

$\mathrm{n}$
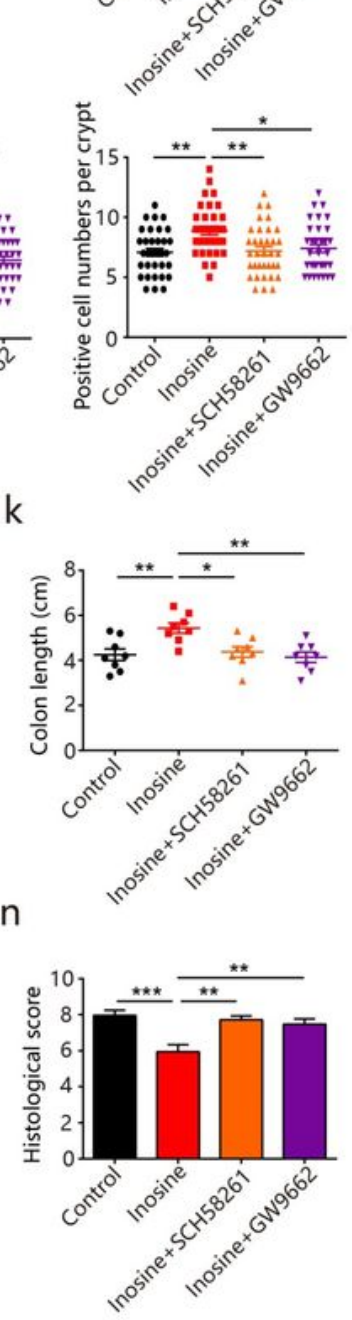

\section{Figure 7}

Inosine protects against dextran sulphate sodium (DSS)-induced colitis though A2AR/PPARy signaling pathway. (a-n) Mice were treated intragastrically with $800 \mathrm{mg} / \mathrm{kg} /$ day of inosine dissolved in PBS at the concentration of $40 \mathrm{mg} / \mathrm{ml}$. PPARy antagonist GW9662 were administered at $3 \mathrm{mg} / \mathrm{kg} / \mathrm{day}$ intragastrically and A2AR antagonist SCH58261 were treated with $2 \mathrm{mg} / \mathrm{kg} /$ day intraperitoneally. Colitis was induced by administering 2.5\% DSS dissolved in drinking water for 7 days. (a) Study design of in 
vivo mouse experiment. (b) Immunofluorescent analysis of PPARy (green) in mouse colonic sections from different mouse groups. Nuclei were stained with DAPI (blue). Scale bar $=100 \mu \mathrm{m}$. (c) Real-time PCR assay for the differentially expressed genes in PPAR signaling pathway $(n=8)$. (d) Crypt height and (e) muscular layer width in the colon of mice from different mouse groups were quantified $(n=12)$. (f) Fecal pellet output and (g) gut transit time from different mouse groups were measured $(n=8)$. (h) Representative alcian blue-stained and Muc2-stained colon sections from different mouse groups. The number of mucusproducing goblet cells and Muc-2 positive goblet cells was quantified $(n=12)$. Scale bar $=100 \mu \mathrm{m}$. (i) Percentage body weight change, (j) diseases activity scores ( $k$ ) colon lengths and (l) intestinal permeability were measured from different mouse groups $(n=8)$. $(m)$ Representative images of hematoxylin and eosin-stained colonic sections and $(n)$ histological scores. Scale bar $=100 \mu \mathrm{m}$. Data are pooled from three independent experiments. Data are mean \pm SEM. ${ }^{\star} P<0.05,{ }^{\star} * P<0.01,{ }^{\star} * \star P<0.001$ and $* * \star * P<0.0001$. For body weight change, a repeated measure two-way analysis of variance (ANOVA) was performed and the rest of the statistics was performed with one-way ANOVA followed by Tukey's multiple comparison's test.

\section{Supplementary Files}

This is a list of supplementary files associated with this preprint. Click to download.

- Additionalfile1.docx

- Additionalfile2.docx 